\title{
Some Exact Solutions of Nonlinear Fin Problem for Steady Heat Transfer in Longitudinal Fin with Different Profiles
}

\author{
M. D. Mhlongo ${ }^{1}$ and R. J. Moitsheki ${ }^{2}$ \\ ${ }^{1}$ Defence, Peace, Safety and Security, Landward Sciences, Council for Scientific and Industrial Research, P.O. Box 395, \\ Pretoria 0001, South Africa \\ ${ }^{2}$ Center for Differential Equations, Continuum Mechanics and Applications, School of Computational and Applied Mathematics, \\ University of the Witwatersrand, Private Bag 3, Johannesburg 2050, South Africa
}

Correspondence should be addressed to R. J. Moitsheki; raseelo.moitsheki@wits.ac.za

Received 19 March 2014; Accepted 2 April 2014; Published 8 May 2014

Academic Editor: Oluwole Daniel Makinde

Copyright (C) 2014 M. D. Mhlongo and R. J. Moitsheki. This is an open access article distributed under the Creative Commons Attribution License, which permits unrestricted use, distribution, and reproduction in any medium, provided the original work is properly cited.

\begin{abstract}
One-dimensional steady-state heat transfer in fins of different profiles is studied. The problem considered satisfies the Dirichlet boundary conditions at one end and the Neumann boundary conditions at the other. The thermal conductivity and heat coefficients are assumed to be temperature dependent, which makes the resulting differential equation highly nonlinear. Classical Lie point symmetry methods are employed, and some reductions are performed. Some invariant solutions are constructed. The effects of thermogeometric fin parameter, the exponent on temperature, and the fin efficiency are studied.
\end{abstract}

\section{Introduction}

Heat transfer through extended surfaces has been studied quite extensively [1], perhaps because of its frequent applications in engineering. Through the process of mathematical modeling, heat transfer problems are reduced to nonlinear differential equations.

Accurate and efficient exact, analytical, and approximate schemes for solving differential equations have been devised through considerable effort, particularly those arising in heat conduction through one-dimensional fin problems (see, e.g., $[2-8])$. The obtained solutions include series solutions $[3,4,7]$, homotopy methods [2], and differential transformation methods (approximate analytical methods) [9]. Few exact solutions exist for one-dimensional problems. In fact, the existing solutions are constructed only for constant thermal conductivity and heat transfer coefficient. Recently, Moitsheki et al. [10] constructed the exact solutions of the one-dimensional fin problem given nonlinear thermal conductivity and heat transfer coefficient. This work has been extended in [11] whereby the introduction of the Kirchhoff transformation linearized the one-dimensional fin problem when heat transfer is a differential consequence of thermal conductivity.

Symmetry methods have been used to analyze the onedimensional fin problems with heat transfer coefficient depending on the spatial variable [12-16]. However, these analyses excluded real-world applications. In recent years, many authors have been interested in the steady-state problems $[2-4,10]$ describing heat flow in one-dimensional longitudinal rectangular fins. The symmetry analysis, in particular, group classification of the unsteady fin problem, has attracted some interests (see, e.g., [12-16]). Recently, Moitsheki and Harley [17] considered fins of various profiles with both heat transfer coefficient and thermal conductivity being given as temperature dependent. An analysis of a steady nonlinear one-dimensional fin of a rectangular profile was given by Moitsheki and Mhlongo [18].

An accurate transient analysis provided insight into the design of fins that failed in steady-state operations but worked well for some operating periods [19]. The transient problem is considered for a fin of arbitrary profile in [20]. However, both thermal conductivity and heat transfer are considered to be constants. Transient response of longitudinal rectangular 


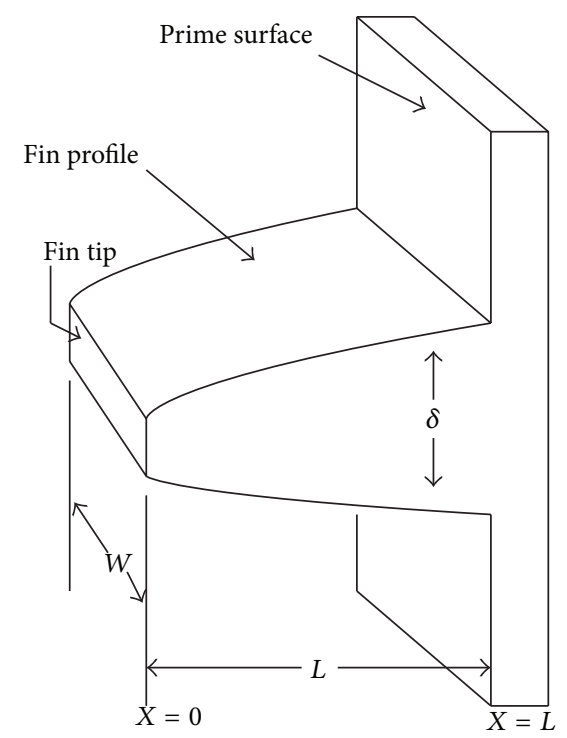

FIGURE 1: Schematic representation of a longitudinal fin of an arbitrary profile.

fins to step change in base temperature and in base heat flow conditions was studied by Mhlongo et al. [21].

In this paper, we determine exact solutions of nonlinear fin problem for steady heat transfer in longitudinal fin of various profiles where the thermal conductivity is related to temperature by a power law. In Section 2, we provide the mathematical formulation of the problem. We determine the exact solution using MAPLE in Section 3. A brief description of symmetry analysis is provided in Section 4. In Section 5, we employ the symmetry techniques to determine, wherever possible, the invariant solutions. Some discussions and concluding remarks are given in Sections 6 and 7, respectively.

\section{Mathematical Models}

We consider a longitudinal one-dimensional fin with a crosssectional area $A_{c}$ as shown in Figure 1. The perimeter of the fin is denoted by $P$ and the length of fin by $L$. The fin is attached to a fixed base surface of temperature $T_{b}$ and extends into a fluid of temperature $T_{a}$. The fin profile is given by the function $F(X)$ and the fin thickness at the base is $\delta_{b}$.

The energy balance for a longitudinal fin is given by

$$
\begin{array}{r}
A_{c} \frac{d}{d X}\left(F(X) K(T) \frac{d T}{d X}\right)=\frac{\delta_{b}}{2} P H(T)\left(T-T_{a}\right), \\
0<X<L,
\end{array}
$$

where $K$ and $H$ are the nonuniform thermal conductivity and heat transfer coefficient depending on the temperature, $T$ is the temperature distribution, $F(X)$ is the fin profile, $t$ is time, and $X$ is the spatial variable. The fin length is measured from the tip to the base. The prescribed boundary conditions are given by (see, e.g., [1])

$$
T(L)=T_{b},\left.\quad \frac{d T}{d X}\right|_{X=0}=0 .
$$

Introducing the dimensionless variables and the dimensionless numbers,

$$
\begin{gathered}
x=\frac{X}{L}, \quad k=\frac{K}{k_{a}}, \quad h=\frac{H}{h_{b}}, \quad M^{2}=\frac{P h_{b} L^{2}}{A_{c} k_{a}}, \\
\theta=\frac{T-T_{a}}{T_{b}-T_{a}}
\end{gathered}
$$

reduce $(1)$ to

$$
\frac{d}{d x}\left(f(x) k(\theta) \frac{d \theta}{d x}\right)=M^{2} h(\theta) \theta, \quad 0<x<1 .
$$

The dimensionless boundary conditions are given by

$$
\theta(1)=1,\left.\quad \frac{d \theta}{d x}\right|_{x=0}=0 .
$$

Here, $M$ is the thermogeometric fin parameter and $\delta$ is the fin thickness, $\theta$ is the dimensionless temperature, $x$ is the dimensionless spatial variable, $f(x)$ is the dimensionless fin profile, $k$ is the dimensionless thermal conductivity, $k_{a}$ is the thermal conductivity of the fin at the ambient temperature, $h$ is the dimensionless heat transfer coefficient, and $h_{b}$ is the heat transfer coefficient at the fin base. For most industrial applications, the heat transfer coefficient may be given as the power law [22]:

$$
H(T)=h_{b}\left(\frac{T-T_{a}}{T_{b}-T_{a}}\right)^{n},
$$

where the exponents $n$ and $h_{b}$ are constants. The constant $n$ may vary between -6.6 and 5 . However, in most practical applications, it lies between -3 and 3 [22]. If the heat transfer coefficient is given by (6), then the hypothetical boundary condition (i.e., insulation) at the tip of the fin is taken into account [22]. If the tip is not assumed to be insulated, then the problem becomes overdetermined (see also [23]). This boundary condition is realized for sufficiently long fins [22]. Besides, the heat transfer through the outermost edge of the fin is negligible compared to that which passes through the side [23]. The exponent $n$ represents laminar film boiling or condensation when $n=-1 / 4$, laminar natural convection when $n=1 / 4$, turbulent natural convection when $n=1 / 3$, nucleate boiling when $n=2$, and radiation when $n=$ 4 , and $n=0$ implies a constant heat transfer coefficient. Exact solutions may be constructed for the steady-state onedimensional differential equation describing temperature distribution in a straight fin when the thermal conductivity is a constant and $n=-1,0,1$, and 2 [22].

The thermal conductivity of the fin may be assumed to vary nonlinearly with the temperature; that is,

$$
K(T)=k_{a}\left(\frac{T-T_{a}}{T_{b}-T_{a}}\right)^{m} .
$$

The one-dimensional heat balance equation is then given by

$$
\frac{d}{d x}\left[f(x) \theta^{m} \frac{d \theta}{d x}\right]=M^{2} \theta^{n+1}, \quad 0<x<1 .
$$


Recently, (8) has been analyzed using the differential transform methods (DTM) [9]. A proposition in the work of Ndlovu and Moitsheki in [9] concluded that $f(x)$, in equations such as (8), needs to be given by an exponential or power law with exponent being strictly 0.5 for DTM to work successfully. Here, we employ basic integration and Lie point symmetry techniques.

2.1. Fin Efficiency. The heat transfer rate from a fin is given by Newton's second law of cooling:

$$
Q=\int_{0}^{L} P H(T)\left(T-T_{a}\right) d X .
$$

Fin efficiency is defined as the ratio of the fin heat transfer rate to the rate that would be if the entire fin was at the base temperature and it is given by (see, e.g., [1])

$$
\eta=\frac{Q}{Q_{\text {ideal }}}=\frac{\int_{0}^{L} P H(T)\left(T-T_{a}\right) d X}{P h_{b} L\left(T_{b}-T_{a}\right)} .
$$

In dimensionless variables, we have

$$
\eta=\int_{0}^{1} \theta^{n+1} d x
$$

2.2. Heat Flux. Heat flux at the base of the fin is given by Fourier's law:

$$
q_{b}=A_{c} K(T) \frac{d T}{d X} .
$$

The total heat flux of the fin is given by [1]

$$
q=\frac{q_{b}}{A_{c} H(T)\left(T-T_{a}\right)} .
$$

In dimensionless variables, we have

$$
q=\frac{1}{B i} \frac{k(\theta)}{h(\theta)} \frac{d \theta}{d x},
$$

where the dimensionless parameter $B i=h_{b} L / k_{a}$ is the Biot number.

\section{Exact Solutions}

In this section, we analyze the governing equation (8), given $m=n$. In this case, $(8)$ is linearizable by a transformation $y=\theta^{n+1}$. Under such a transformation, (8) becomes

$$
\frac{d}{d x}\left[f(x) \frac{d y}{d x}\right]-(n+1) M^{2} y=0,
$$

and the boundary conditions transform to

$$
y(1)=1, \quad y^{\prime}(0)=0 .
$$

Equation (15) is analyzed for various situations in the next sections and all the solutions in illustrative examples satisfy both the Dirichlet and the Neumann boundary conditions.

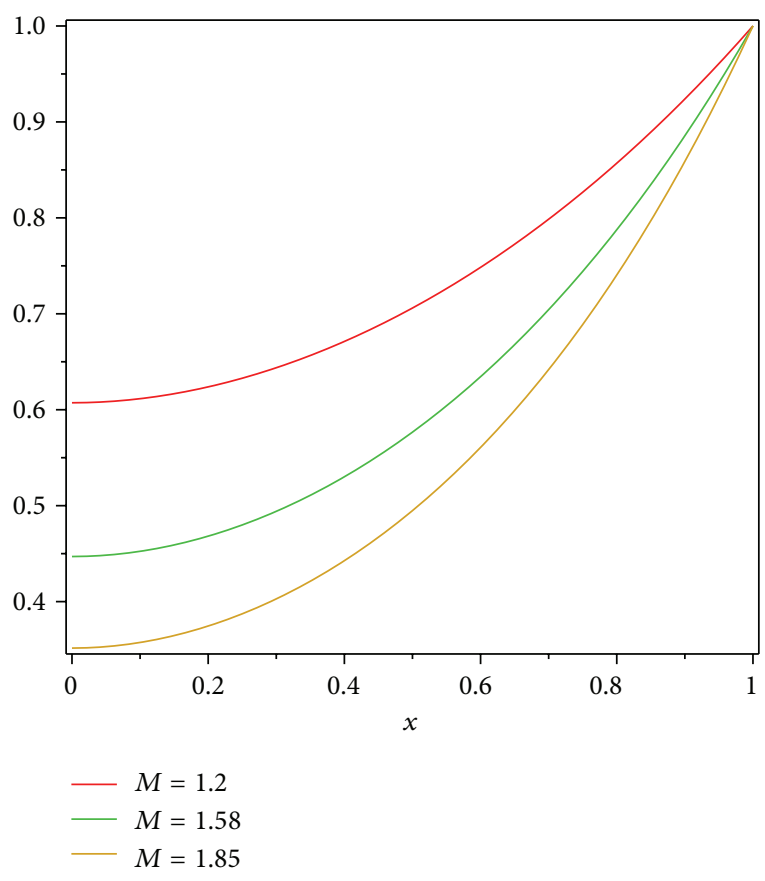

FigURE 2: Temperature distribution for the profile $f(x)=\sqrt{x+1}$ given in (18) in a fin with varying values of $M$.

3.1. Case: $n>-1$ with $m=n \neq-1$. As an illustration, we use two examples, and the rest of the exact solutions are listed in Tables 1 and 2.

Example 1. Given $f(x)=\sqrt{x+1}$, (15) becomes

$$
(x+1) y^{\prime \prime}+y^{\prime}-(n+1) M^{2} \sqrt{x+1}=0,
$$

with solution

$\theta$

$$
=\left[(x+1)^{1 / 4} J_{1 / 3}\left(\beta\left(\frac{x+1}{2}\right)^{3 / 4}\right)\left(\frac{Y}{J_{1 / 3}(\beta)}-Y-1\right)\right]^{1 /(n+1)},
$$

where $\beta=(4 M i \sqrt{n+1}) / 3$ and $\gamma=\sqrt[4]{8}$, with $i=\sqrt{-1}$. Consider the following:

$$
\begin{aligned}
Y=\frac{1}{2}( & \left.Y_{1 / 3}\left(\frac{\beta}{\gamma}\right)+2 Y_{4 / 3}(\beta) M \sqrt{n+1}\right) J_{1 / 3}(\beta \gamma) \\
& \times\left(-J_{1 / 3}(\beta) Y_{1 / 3}(\beta \gamma)+2 Y_{1 / 3}(\beta \gamma) J_{4 / 3}(\beta) M \sqrt{n+1}\right. \\
& \left.+Y_{1 / 3}(\beta) J_{1 / 3}(\beta \gamma)-2 J_{1 / 3}(\beta \gamma) Y_{4 / 3}(\beta) M \sqrt{n+1}\right)^{-1} .
\end{aligned}
$$

The efficiency is given by

$$
\begin{aligned}
\eta=\int_{0}^{1}[ & (x+1)^{1 / 4} J_{1 / 3}\left(\beta\left(\frac{x+1}{2}\right)^{3 / 4}\right) \\
& \left.\times\left(\frac{Y}{J_{1 / 3}(\beta)}-Y-1\right)\right] d x .
\end{aligned}
$$


TABLE 1: Solution for $n>-1$ with $m=n \neq-1$.

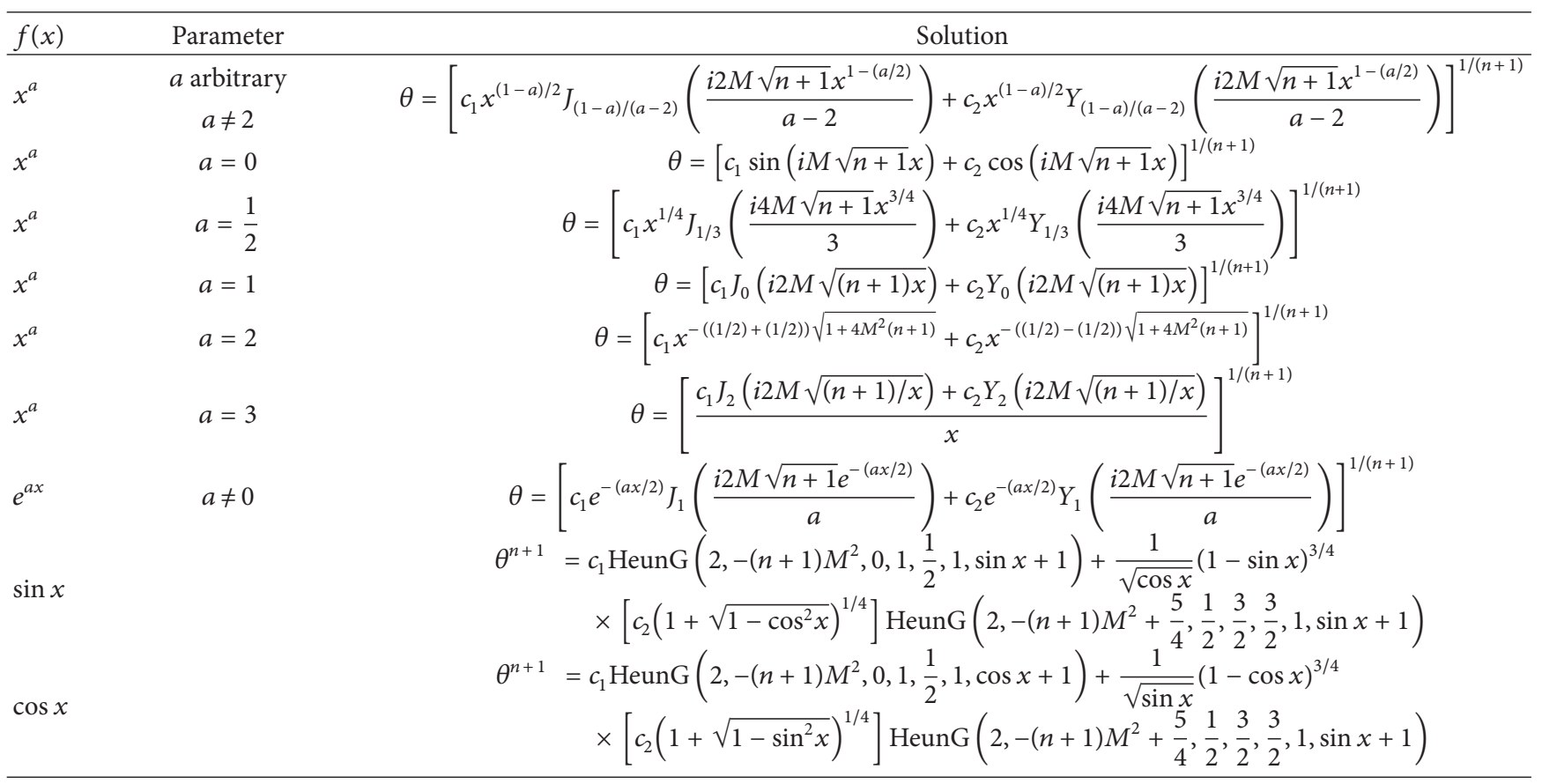

TABLE 2: Modified general solution for $f(\mathscr{X})$ where $\mathscr{X}=x+1, n>-1$, and $m=n \neq-1$.

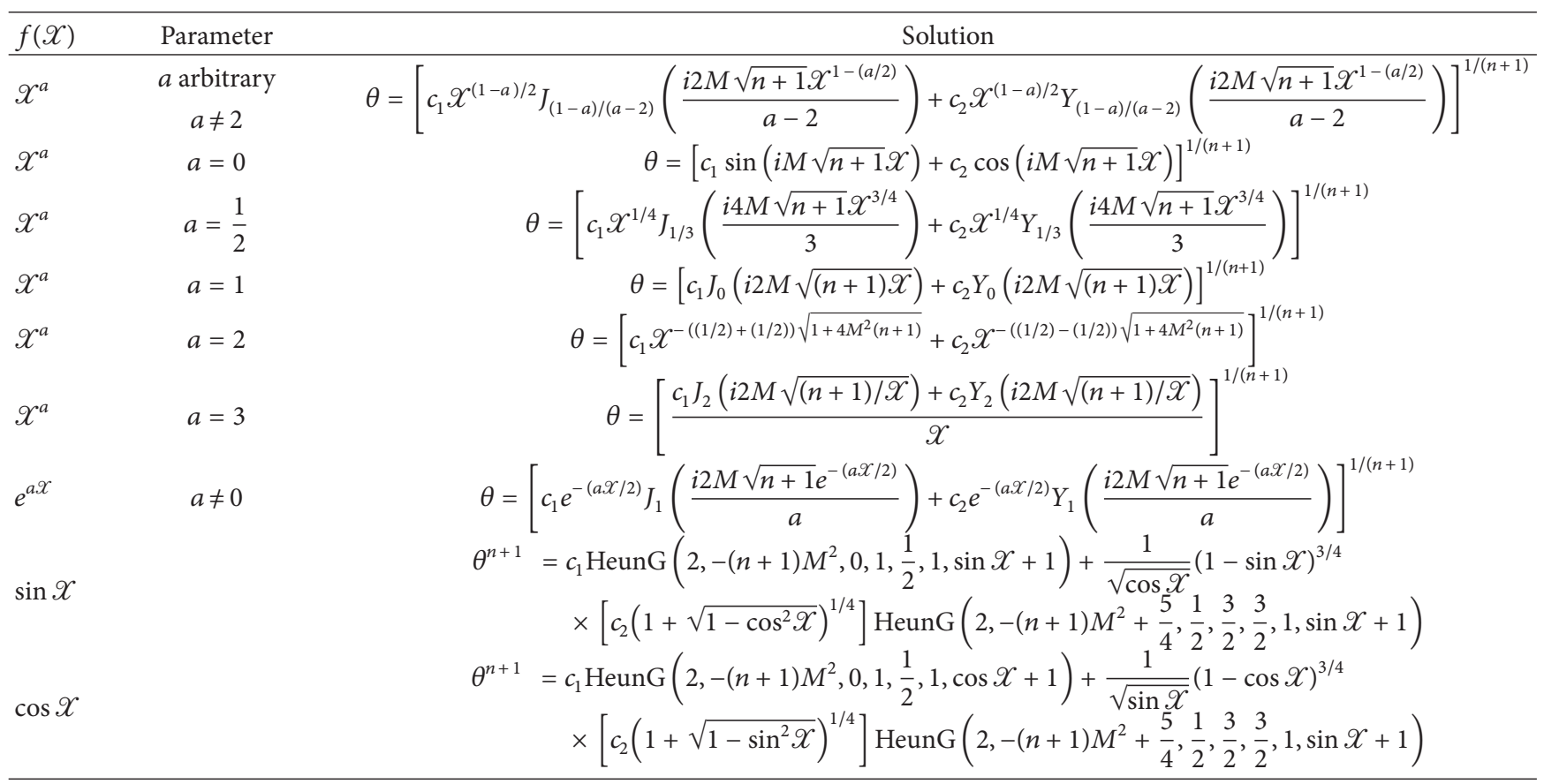

The temperature distribution along the surface for this profile is depicted in Figures 2 and 3. The fin efficiency as function of the thermogeometric parameter is shown in Figure 4.

Example 2. In case of $f(x)=(x+1)^{3}$, then (15) is transformed into

$$
(x+1)^{3} y^{\prime \prime}+3(x+1)^{2} y^{\prime}-(n+1) M^{2}=0
$$

which is solved by Bessel functions, and the solution in terms of original variables is

$$
\theta=\left[\frac{J_{2}(\alpha / \sqrt{x+1}) J}{J_{2}(\alpha / \sqrt{2})(x+1)}-\frac{Y_{2}(\alpha / \sqrt{x+1})}{Y_{2}(\alpha / \sqrt{2})(x+1)}(J-2)\right]^{1 /(n+1)},
$$


TABLE 3: Solution for $n<-1$ with $m=n \neq-1$.

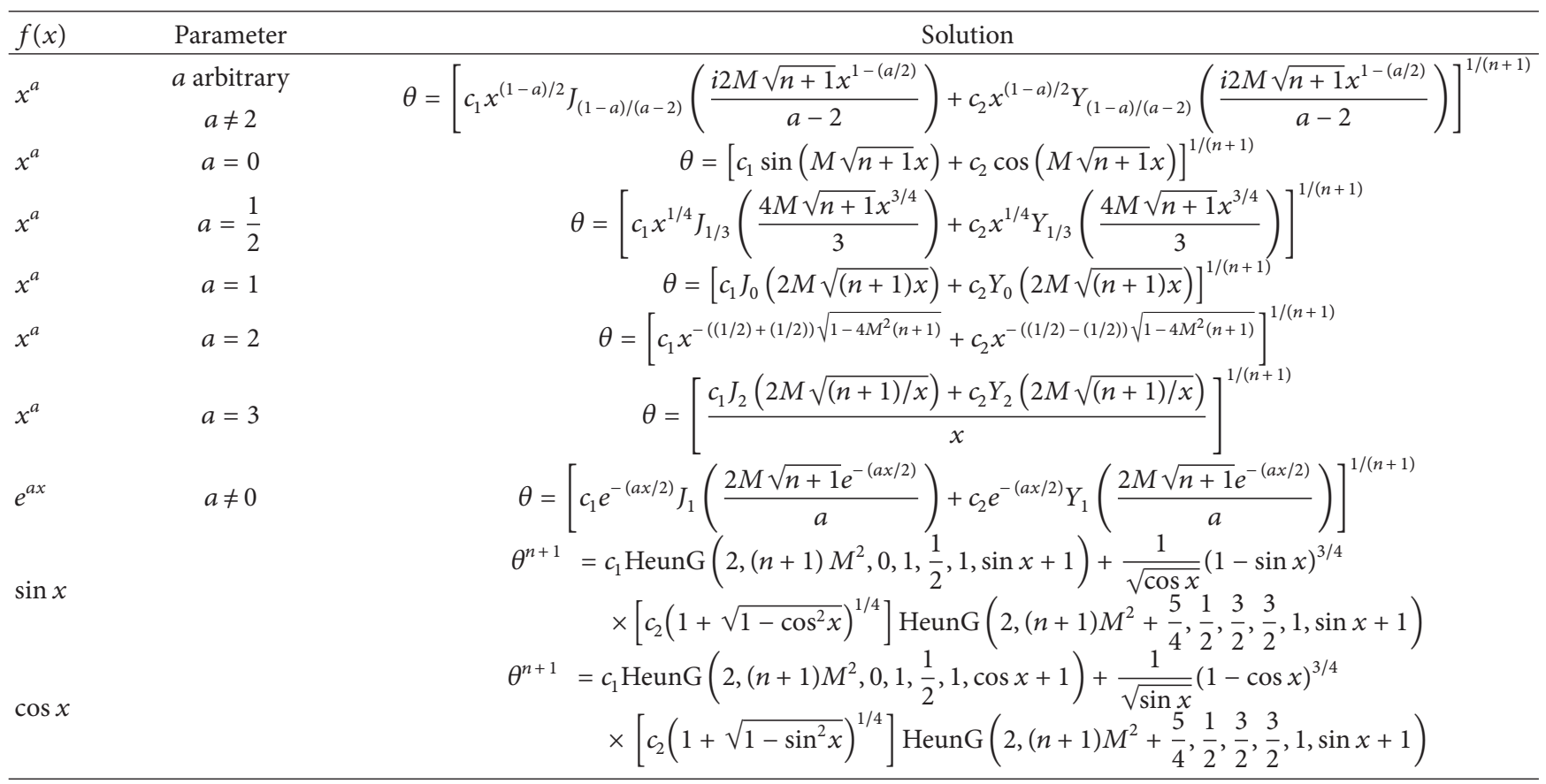

TABLE 4: Modified solution for $f(\mathscr{X})$ where $\mathscr{X}=x+1, n<-1$, and $m=n \neq-1$.

\begin{tabular}{|c|c|c|}
\hline$f(x)$ & Parameter & Solution \\
\hline \multirow{2}{*}{$\mathscr{X}^{a}$} & $a$ arbitrary & $\left.\left(i 2 M \sqrt{n+1} X^{1-(a / 2)}\right) \quad\left(i 2 M \sqrt{n+1} X^{1-(a / 2)}\right)\right]^{1 /}$ \\
\hline & $a \neq 2$ & 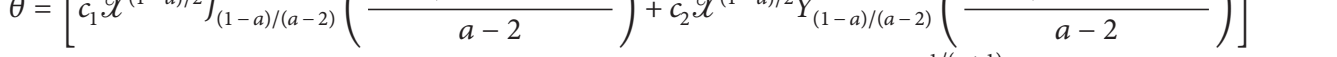 \\
\hline $\mathscr{X}^{a}$ & $a=0$ & $\theta=\left[c_{1} \sin (M \sqrt{n+1} X)+c_{2} \cos (M \sqrt{n+1} X)\right]^{1 /(n+1)}$ \\
\hline $\mathscr{X}^{a}$ & $a=\frac{1}{2}$ & $\theta=\left[c_{1} X^{1 / 4} J_{1 / 3}\left(\frac{4 M \sqrt{n+1} X^{3 / 4}}{3}\right)+c_{2} X^{1 / 4} Y_{1 / 3}\left(\frac{4 M \sqrt{n+1} X^{3 / 4}}{3}\right)\right]^{1 /(n+1)}$ \\
\hline $\mathscr{X}^{a}$ & $a=1$ & $\theta=\left[c_{1} J_{0}(2 M \sqrt{(n+1) X})+c_{2} Y_{0}(2 M \sqrt{(n+1) \mathscr{X}})\right]^{1 /(n+1)}$ \\
\hline $\mathscr{X}^{a}$ & $a=2$ & $\theta=\left[c_{1} \mathscr{X}^{-((1 / 2)+(1 / 2))} \sqrt{1-4 M^{2}(n+1)}+c_{2} \mathscr{X}^{-((1 / 2)-(1 / 2))} \sqrt{1-4 M^{2}(n+1)}\right]^{1 /(n+1)}$ \\
\hline $\mathscr{X}^{a}$ & $a=3$ & $\theta=\left[\frac{c_{1} J_{2}(2 M \sqrt{(n+1) / \mathscr{X}})+c_{2} Y_{2}(2 M \sqrt{(n+1) / \mathscr{X}})}{\mathscr{X}}\right]^{1 /(}$ \\
\hline$e^{a x}$ & $a \neq 0$ & $\theta=\left[c_{1} e^{-(a x / 2)} J_{1}\left(\frac{2 M \sqrt{n+1} e^{-(a x / 2)}}{a}\right)+c_{2} e^{-(a x / 2)} Y_{1}\left(\frac{2 M \sqrt{n+1} e^{-(a x / 2)}}{a}\right)\right]^{1 /(n}$ \\
\hline $\sin X$ & & $\begin{aligned} \theta^{n+1}= & c_{1} \operatorname{HeunG}\left(2,(n+1) M^{2}, 0,1, \frac{1}{2}, 1, \sin \mathscr{X}+1\right)+\frac{1}{\sqrt{\cos \mathscr{X}}}(1-\sin \mathscr{X})^{3 / 4} \\
& \times\left[c_{2}\left(1+\sqrt{1-\cos ^{2} \mathscr{X}}\right)^{1 / 4}\right] \operatorname{HeunG}\left(2,(n+1) M^{2}+\frac{5}{4}, \frac{1}{2}, \frac{3}{2}, \frac{3}{2}, 1, \sin \mathscr{X}+1\right)\end{aligned}$ \\
\hline $\cos \mathscr{X}$ & & $\begin{aligned} \theta^{n+1}= & c_{1} \operatorname{HeunG}\left(2,(n+1) M^{2}, 0,1, \frac{1}{2}, 1, \cos X+1\right)+\frac{1}{\sqrt{\sin \mathscr{X}}}(1-\cos X)^{3 / 4} \\
& \times\left[c_{2}\left(1+\sqrt{1-\sin ^{2} \mathscr{X}}\right)^{1 / 4}\right] \operatorname{HeunG}\left(2,(n+1) M^{2}+\frac{5}{4}, \frac{1}{2}, \frac{3}{2}, \frac{3}{2}, 1, \sin X+1\right)\end{aligned}$ \\
\hline
\end{tabular}

where $\alpha=2 M i \sqrt{n+1}$ and

$$
J=\frac{-2 Y_{1}(\alpha) J_{2}(\alpha / \sqrt{2})}{Y_{2}(\alpha / \sqrt{2}) J_{1}(\alpha)-J_{2}(\alpha / \sqrt{2}) Y_{1}(\alpha)}
$$

The efficiency is given by

$$
\eta=\int_{0}^{1}\left[\frac{J_{2}(\alpha / \sqrt{x+1}) J}{J_{2}(\alpha / \sqrt{2})(x+1)}-\frac{Y_{2}(\alpha / \sqrt{x+1})}{Y_{2}(\alpha / \sqrt{2})(x+1)}(J-2)\right] d x
$$


TABLE 5: Solution for $m=n=-1$.

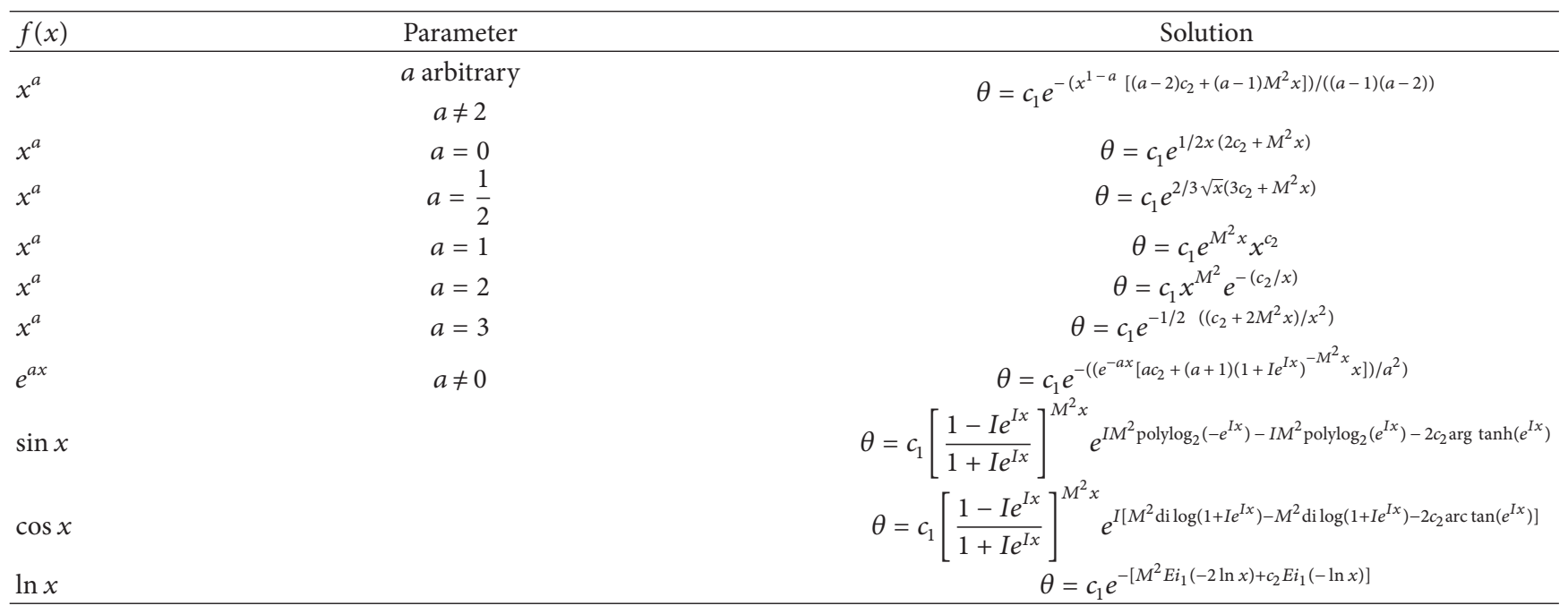

TABle 6: Modified general solution for $f(\mathscr{X})$ where $\mathscr{X}=x+1$ and $m=n=-1$.

\begin{tabular}{|c|c|c|}
\hline$f(x)$ & Parameter & Solution \\
\hline $\mathscr{X}^{a}$ & $\begin{array}{l}a \text { arbitrary } \\
a \neq 2\end{array}$ & $\theta=c_{1} e^{-\left(X^{1-a}\left[(a-2) c_{2}+(a-1) M^{2} X\right]\right) /((a-1)(a-2))}$ \\
\hline $\mathscr{X}^{a}$ & $\begin{array}{l}a \neq 2 \\
a=0\end{array}$ & $\theta=c_{1} e^{1 / 2 X\left(2 c_{2}+M^{2} X\right)}$ \\
\hline $\mathscr{X}^{a}$ & $a=\frac{1}{2}$ & $\theta=c_{1} e^{2 / 3 \sqrt{X}\left(3 c_{2}+M^{2} X\right)}$ \\
\hline $\mathscr{X}^{a}$ & $a=1$ & $\theta=c_{1} e^{M^{2} X} X^{c_{2}}$ \\
\hline$x^{a}$ & $a=2$ & $\theta=c_{1} \mathscr{X}^{M^{2}} e^{-\left(c_{2} / \mathscr{X}\right)}$ \\
\hline$x^{a}$ & $a=3$ & $\theta=c_{1} e^{-1 / 2\left(\left(c_{2}+2 M^{2} X\right) / X^{2}\right)}$ \\
\hline$e^{a x}$ & $a \neq 0$ & $\theta=c_{1} e^{-\left(e^{-a x}\left[a c_{2}+(a+1)\left(1+I e^{I X}\right)^{-M^{2} X} X\right]\right) / a^{2}}$ \\
\hline $\sin x$ & & $\theta=c_{1}\left[\frac{1-I e^{I X}}{1+I e^{I X}}\right]^{M^{2} X} e^{I M^{2} \text { poly } \log _{2}\left(-e^{I X}\right)-I M^{2} \text { poly } \log _{2}\left(e^{I X}\right)-2 c_{2} \arg \tanh \left(e^{I X}\right)}$ \\
\hline $\cos \mathscr{X}$ & & $\theta=c_{1}\left[\frac{1-I e^{I X}}{1+I e^{I X}}\right]^{M^{2} X} \quad e^{I\left[M^{2} \operatorname{dil} \log \left(1+I e^{I X}\right)-M^{2} \operatorname{di} \log \left(1+I e^{I X}\right)-2 c_{2} \arctan \left(e^{I X}\right)\right]}$ \\
\hline $\ln x$ & & $\theta=c_{1} e^{-\left[M^{2} E i_{1}(-2 \ln X)+c_{2} E i_{1}(-\ln X)\right]}$ \\
\hline
\end{tabular}

The temperature distribution along the surface for this profile is depicted in Figures 5 and 6. The fin efficiency as function of the thermogeometric fin parameter is shown in Figure 7.

3.2. Case: $n<-1$ with $m=n$. We will use two examples as in the previous case; the rest of the solutions are listed in Tables 3 and 4.

Example 3. Starting with $f(x)=x^{2},(15)$ in its changed form will be

$$
x^{2} y^{\prime \prime}+2 x y^{\prime}+(n+1) M^{2}=0
$$

with solution

$$
\theta=x^{(1 / 2(n+1))\left(\sqrt{1-4(n+1) M^{2}}-1\right)} .
$$

The efficiency is given by

$$
\begin{aligned}
\eta & =\int_{0}^{1} x^{(1 / 2)\left(\sqrt{1-4(n+1) M^{2}}-1\right)} d x \\
& =\frac{2}{\sqrt{1-4(n+1) M^{2}}+1+2 n} .
\end{aligned}
$$

Example 4. We consider $f(x)=\sqrt{x+1}$ as the second example. This transforms (15) into

$$
(x+1) y^{\prime \prime}+y^{\prime}+(n+1) M^{2} \sqrt{x+1}=0
$$

with solution

$$
\theta=\left[(x+1)^{1 / 4} J_{1 / 3}\left(\beta\left(\frac{x+1}{2}\right)^{3 / 4}\right)\left(\frac{Y}{J_{1 / 3}(\beta)}-Y-1\right)\right]^{1 /(n+1)},
$$


TABLE 7: Symmetries for $m=n=-1, f(x)=x^{a}$.

\begin{tabular}{|c|c|c|}
\hline$f(x)$ & Symmetries & \\
\hline 1 & $\begin{array}{c}X_{1}=\frac{\partial}{\partial \theta}, X_{2}=2 x^{2} \frac{\partial}{\partial x}+\left(2 \ln \theta+x^{2} M^{2}\right) \theta \frac{\partial}{\partial \theta} \\
X_{4}=2\left(x^{2} M^{2}-2 \ln \theta\right) x \frac{\partial}{\partial x}+\left(x^{4} M^{4}-4 \ln ^{2} \theta\right) \theta \frac{\partial}{\partial \theta} \\
X_{6}=\left(3 x^{2} M^{2}-2 \ln \theta\right) \frac{\partial}{\partial x}+2 x^{3} M^{4} \theta \frac{\partial}{\partial \theta}\end{array}$ & $\begin{array}{l}X_{3}=x \frac{\partial}{\partial x}+x^{2} M^{2} \theta \frac{\partial}{\partial \theta} \\
X_{5}=\left(2 \ln \theta-x^{2} M^{2}\right) \theta \frac{\partial}{\partial \theta} \\
X_{7}=-x \theta \frac{\partial}{\partial \theta}, X_{8}=-\theta \frac{\partial}{\partial \theta}\end{array}$ \\
\hline$\sqrt{x}$ & $\begin{array}{c}X_{1}=-\theta \frac{\partial}{\partial \theta}, X_{2}=-4 x \sqrt{x} \frac{\partial}{\partial x}-\left(\frac{8}{3} x^{2} M^{2}=2 \sqrt{x} \ln \theta\right) \theta \frac{\partial}{\partial \theta} \\
X_{4}=\left(\sqrt{x} \ln \theta-\frac{2}{3} x^{2} M^{2}\right) \frac{\partial}{\partial x}+\left(x \ln \theta-\frac{2}{3} x^{2} \sqrt{x} M^{2}\right) M^{2} \theta \frac{\partial}{\partial \theta} \\
X_{6}=2 \sqrt{x} \theta \frac{\partial}{\partial \theta}, \\
X_{7}=-\frac{1}{\sqrt{x}} \frac{\partial}{\partial x}-x M^{2} \theta \frac{\partial}{\partial \theta}-\left(\frac{1}{2} \ln ^{2} \theta-\frac{4}{9} x^{3} M^{4}+\frac{1}{3} x \sqrt{x} M^{2} \ln \theta\right) \theta \frac{\partial}{\partial \theta}\end{array}$ & $\begin{aligned} X_{3} & =x \frac{\partial}{\partial x}+x \sqrt{x} M^{2} \theta \frac{\partial}{\partial \theta} \\
X_{5} & =\frac{\left(3 \sqrt{x} \ln \theta-2 x^{2} M^{2}\right)}{3 \sqrt{x}} \theta \frac{\partial}{\partial \theta} \\
X_{8} & =\left(\frac{2}{3} x \sqrt{x} M^{2}-\ln \theta\right) x \frac{\partial}{\partial x}\end{aligned}$ \\
\hline$x$ & $\begin{array}{c}X_{1}=-x^{2} \ln x \frac{\partial}{\partial x}+\left(x M^{2}-x \ln x M^{2}-\ln \theta\right) \theta \ln x \frac{\partial}{\partial \theta} \\
X_{3}=\left(\ln \theta-x M^{2}\right) x \frac{\partial}{\partial x}+\left(\ln \theta-M^{2} x\right) x \theta M^{2} \frac{\partial}{\partial \theta} \\
X_{5}=-\theta \frac{\partial}{\partial \theta}, X_{6}=\theta \ln x \frac{\partial}{\partial \theta}, \\
X_{7}=\left(\ln \theta-x M^{2}\right) \theta \frac{\partial}{\partial \theta}-\theta\left(\ln ^{2} \theta+\ln \theta \ln x M^{2} x-2 x \ln \theta M^{2}-x^{2} M^{4} \ln x+x^{2} M^{4}\right) \frac{\partial}{\partial \theta}\end{array}$ & $\begin{array}{c}X_{2}=x \ln x \frac{\partial}{\partial x}+x \ln x M^{2} \theta \frac{\partial}{\partial \theta} \\
X_{4}=-x \frac{\partial}{\partial x}-x M^{2} \theta \frac{\partial}{\partial \theta} \\
X_{8}=-\left(\ln \theta-x M^{2}\right) x \ln x \frac{\partial}{\partial x}\end{array}$ \\
\hline$x^{2}$ & $\begin{array}{l}X_{1}=-x \frac{\partial}{\partial x}, X_{2}=-\frac{\partial}{\partial x}+\left(\frac{\ln \theta-\ln x M^{2}-M^{2}}{x}\right) \theta \frac{\partial}{\partial \theta} \\
X_{4}=x^{2}\left(\ln x M^{2}-\ln \theta\right) \frac{\partial}{\partial x}+x \theta\left(\ln x M^{2}-\ln \theta\right) M^{2} \frac{\partial}{\partial x} \\
X_{6}=x^{2}\left(M^{2}-\ln x-\ln \theta\right) \frac{\partial}{\partial x}+\theta\left(\ln \theta-M^{2} \ln x\right)^{2} \frac{\partial}{\partial \theta}\end{array}$ & $\begin{array}{l}X_{3}=-x^{2} \frac{\partial}{\partial x}-x \theta M^{2} \frac{\partial}{\partial \theta} \\
X_{5}=\left(\ln \theta-M^{2} \ln x\right) \theta \frac{\partial}{\partial \theta} \\
X_{7}=-\frac{\theta}{x} \frac{\partial}{\partial \theta}, X_{8}=-\theta \frac{\partial}{\partial \theta}\end{array}$ \\
\hline$e^{a x}$ & $\begin{array}{c}X_{1}=-\theta \frac{\partial}{\partial \theta}, X_{2}=-\frac{\theta}{a e^{a x}} \frac{\partial}{\partial \theta}, X_{3}=-\frac{e^{a x}}{a} \frac{\partial}{\partial x}-\frac{x \theta M^{2}}{a} \frac{\partial}{\partial \theta} \\
X_{5}=\frac{1}{a^{2} e^{a x}} \frac{\partial}{\partial x}+\left(a^{2} e^{a x} \ln x+M^{2}\right) \theta \frac{\partial}{\partial \theta} \\
X_{7}=\left(\frac{a e^{a x} \ln \theta+x M^{2}}{a^{2}}\right) \theta \frac{\partial}{\partial x}+x \theta M^{2}\left(\frac{a^{2} e^{a x} \ln \theta+x M^{2}}{a^{2} e^{a x}}\right) \theta \frac{\partial}{\partial \theta} \\
+\left(\frac{a^{3} e^{2 a x} \ln ^{2} \theta+a^{2} x e^{a x} M^{2} \ln \theta+a e^{a x} M^{2} \ln \theta+x M^{4}}{a e^{a x}}\right) \theta \frac{\partial}{\partial \theta}\end{array}$ & $\begin{array}{c}X_{4}=\frac{\partial}{\partial x}+(a x+1) M^{2} \frac{\partial}{\partial \theta} \\
X_{6}=\left(\frac{a^{2} e^{a x} \ln \theta+(a x+1) M^{2}}{a^{2} e^{a x}}\right) \theta \frac{\partial}{\partial \theta} \\
X_{8}=\left(\frac{a e^{a x} \ln \theta+x M^{2}}{a e^{a x}}\right) \theta \frac{\partial}{\partial x}\end{array}$ \\
\hline
\end{tabular}

where $\beta=4 M \sqrt{n+1} / 3$ and $\gamma=\sqrt[4]{8}$. Consider the following:

$$
\begin{aligned}
Y=\frac{1}{2}( & \left.Y_{1 / 3}\left(\frac{\beta}{\gamma}\right)+2 Y_{4 / 3}(\beta) M \sqrt{n+1}\right) J_{1 / 3}(\beta \gamma) \\
\times & \left(-J_{1 / 3}(\beta) Y_{1 / 3}(\beta \gamma)+2 Y_{1 / 3}(\beta \gamma) J_{4 / 3}(\beta) M \sqrt{n+1}\right. \\
& \left.+Y_{1 / 3}(\beta) J_{1 / 3}(\beta \gamma)-2 J_{1 / 3}(\beta \gamma) Y_{4 / 3}(\beta) M \sqrt{n+1}\right)^{-1} .
\end{aligned}
$$

The efficiency is given by

$$
\begin{aligned}
\eta=\int_{0}^{1}[ & (x+1)^{1 / 4} J_{1 / 3}\left(\beta\left(\frac{x+1}{2}\right)^{3 / 4}\right) \\
& \left.\times\left(\frac{Y}{J_{1 / 3}(\beta)}-Y-1\right)\right] d x .
\end{aligned}
$$

3.3. Case: $m=n=-1$. The governing equation (8) becomes

$$
\frac{d}{d x}\left[f(x) \theta^{-1} \frac{d \theta}{d x}\right]=M^{2} \text {. }
$$

After simplification, (32) becomes

$$
\frac{d \theta}{\theta}=\left[\frac{M^{2} x+c_{1}}{f(x)}\right] d x .
$$

The solutions to (33) for various $f(x)$ are given in Table 9.

Example 5. $f(x)=x^{3}$ is considered as an example, and the solution is

$$
\theta=e^{M^{2}(1-(1 / x))}
$$

and satisfying boundary conditions

$$
\theta(1)=1, \quad \lim _{x \rightarrow 0} \frac{d \theta}{d x}=0 .
$$


TABLE 8: Symmetries for $m \neq n, n \neq-1$ and various $f(x)$.

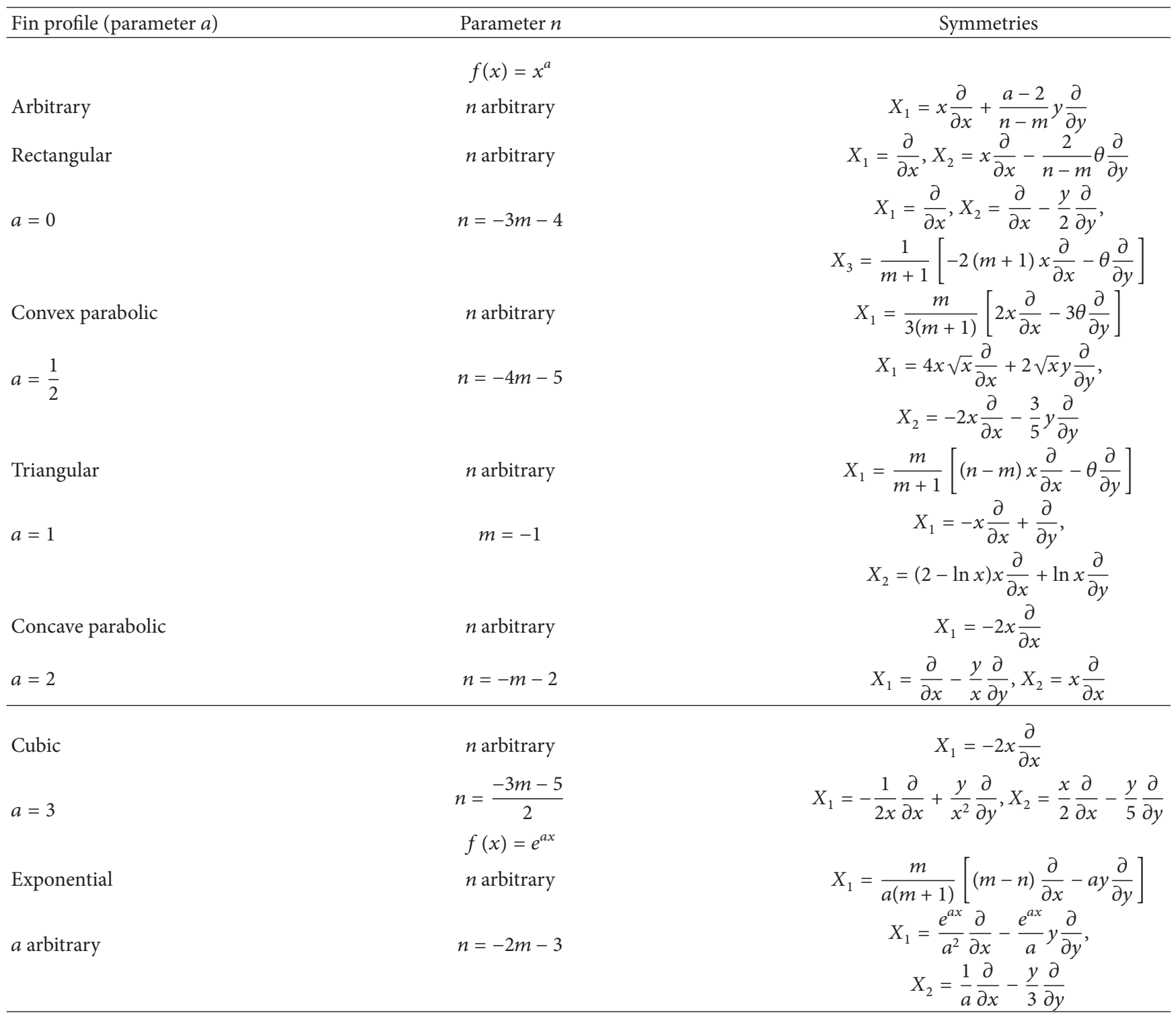

The corresponding fin efficiency is given by

$$
\eta=\int_{0}^{1} e^{M^{2}(1-(1 / x))} d x
$$

Other exact solutions are listed in Tables 5, 6, and 7.

\subsection{Case: $m \neq n$}

Example 6. We consider $f(x)=1$ and $n=-3 m-4$ and let $y=\theta^{m+1}$, which transforms the governing equation (8) into

$$
y^{\prime \prime}=(m+1) M^{2} y^{-3}
$$

By direct integration using Polyanin and Zaitsev [24], we get

$$
x=\int\left(\frac{2 c_{1} y^{2}-2(m+1) M^{2}}{2 y^{2}}\right)^{-1 / 2} d y+c_{2},
$$

which yields

$$
x=\sqrt{\frac{c_{1} y^{2}-(m+1) M^{2}}{c_{1}^{2}}}+c_{2} .
$$

Using the boundary conditions (16), we get the solution

$$
y=\sqrt{\frac{(1 / 2)\left[1+\sqrt{1-4(m+1) M^{2}}\right]^{2} x^{2}+2(m+1) M^{2}}{1+\sqrt{1-4(m+1) M^{2}}}} .
$$

In terms of the original variables, we have

$$
\begin{aligned}
\theta=\left[\frac{1}{2}\left[1+\sqrt{1-4(m+1) M^{2}}\right]^{2} x^{2}+2(m+1) M^{2}\right. \\
\left.\quad \times\left(1+\sqrt{1-4(m+1) M^{2}}\right)^{-1}\right]^{1 / 2(m+1)} .
\end{aligned}
$$


Table 9: Modified symmetries for $f(\mathscr{X})$ where $\mathscr{X}=x+1$ and $m \neq n, n \neq-1$.

\begin{tabular}{|c|c|c|}
\hline Fin profile (parameter $a$ ) & Parameter $n$ & Symmetries \\
\hline & $f(X)=\mathscr{X}^{a}$ & \\
\hline Arbitrary & $n$ arbitrary & $X_{1}=x \frac{\partial}{\partial x}+\frac{a-2}{n-m} y \frac{\partial}{\partial y}$ \\
\hline Rectangular & $n$ arbitrary & $X_{1}=\frac{\partial}{\partial x}, X_{2}=x \frac{\partial}{\partial x}-\frac{2}{n-m} \theta \frac{\partial}{\partial y}$ \\
\hline$a=0$ & $n=-3 m-4$ & $\begin{array}{c}X_{1}=\frac{\sigma}{\partial x}, X_{2}=\frac{\sigma}{\partial x}-\frac{y}{2} \frac{\sigma}{\partial y}, \\
X_{3}=\frac{1}{m+1}\left[-2(m+1) x \frac{\partial}{\partial x}-\theta \frac{\partial}{\partial y}\right]\end{array}$ \\
\hline Convex parabolic & $n$ arbitrary & $X_{1}=\frac{m}{3(m+1)}\left[2 X \frac{\partial}{\partial x}-3 \theta \frac{\partial}{\partial y}\right]$ \\
\hline$a=\frac{1}{2}$ & $n=-4 m-5$ & $\begin{array}{c}X_{1}=4 \mathscr{X} \sqrt{x} \frac{\partial}{\partial x}+2 \sqrt{x} y \frac{\partial}{\partial y} \\
X_{2}=-2 x \frac{\partial}{\partial x}-\frac{3}{5} y \frac{\partial}{\partial y}\end{array}$ \\
\hline Triangular & $n$ arbitrary & $X_{1}=\frac{m}{m+1}\left[(n-m) X \frac{\partial}{\partial x}-\theta \frac{\partial}{\partial y}\right]$ \\
\hline$a=1$ & $m=-1$ & $\begin{array}{c}X_{1}=-\mathscr{X} \frac{\partial}{\partial x}+\frac{\partial}{\partial y}, \\
X_{2}=(2-\ln \mathscr{X}) \mathscr{X} \frac{\partial}{\partial x}+\ln \mathscr{X} \frac{\partial}{\partial y}\end{array}$ \\
\hline Concave parabolic & $n$ arbitrary & $X_{1}=-2 \mathscr{X} \frac{\partial}{\partial x}$ \\
\hline$a=2$ & $n=-m-2$ & $X_{1}=\frac{\partial}{\partial x}-\frac{y}{x} \frac{\partial}{\partial y}, X_{2}=x \frac{\partial}{\partial x}$ \\
\hline Cubic & $n$ arbitrary & $X_{1}=-2 x \frac{\partial}{\partial x}$ \\
\hline$a=3$ & $n=\frac{-3 m-5}{2}$ & $X_{1}=-\frac{1}{2 \mathscr{X}} \frac{\partial}{\partial x}+\frac{y}{\mathscr{X}^{2}} \frac{\partial}{\partial y}, X_{2}=\frac{\mathscr{X}}{2} \frac{\partial}{\partial x}-\frac{y}{5} \frac{\partial}{\partial y}$ \\
\hline & $f(\mathscr{X})=e^{a \mathscr{X}}$ & \\
\hline Exponential & $n$ arbitrary & $X_{1}=\frac{m}{a(m+1)}\left[(m-n) \frac{\partial}{\partial x}-a y \frac{\partial}{\partial y}\right]$ \\
\hline$a$ arbitrary & $n=-2 m-3$ & $\begin{array}{c}X_{1}=\frac{}{a^{2}} \frac{\partial}{\partial x}-\frac{}{a} y \frac{\partial}{\partial y} \\
X_{2}=\frac{1}{a} \frac{\partial}{\partial x}-\frac{y}{3} \frac{\partial}{\partial y}\end{array}$ \\
\hline
\end{tabular}

The corresponding fin efficiency is given by

$$
\begin{aligned}
\eta=\int_{0}^{1}[ & \frac{1}{2}\left[1+\sqrt{1-4(m+1) M^{2}}\right]^{2} x^{2}+2(m+1) M^{2} \\
& \left.\times\left(1+\sqrt{1-4(m+1) M^{2}}\right)^{-1}\right]^{1 / 2} d x
\end{aligned}
$$

Other exact solutions are listed in Tables 8 and 9.

\section{Lie Point Symmetry Analysis}

The theory and applications of symmetry analysis may be found in excellent text such as those of [25-29]. In brief, the symmetry of a differential equation is an invertible transformation of dependent and independent variables which leave the form of the equation in question unchanged [30]. To determine the symmetry for the governing equation (8), one may seek the transformations of the following form:

$$
\begin{aligned}
& \bar{x}=x+\epsilon \xi(x, \theta)+O\left(\epsilon^{2}\right), \\
& \bar{\theta}=\theta+\eta \xi(x, \theta)+O\left(\epsilon^{2}\right) .
\end{aligned}
$$

The infinitesimal transformations $(43)$ act on the $(x, \theta)$ space with the corresponding infinitesimal generator

$$
X=\xi(x, \theta) \partial_{x}+\eta(x, \theta) \partial_{\theta},
$$

which leaves the governing equation invariant. We will then apply the boundary condition to the obtained invariant solutions. The action of $X$ is extended in the governing equation through the second prolongation given by

$$
X^{[2]}=X+\zeta_{x} \partial_{\theta^{\prime}}+\zeta_{x x} \partial_{\theta^{\prime \prime}}
$$

where

$$
\begin{gathered}
\zeta_{x}=D_{x}(\eta)-\theta^{\prime} D_{x}(\xi), \\
\zeta_{x x}=D_{x}\left(\zeta_{x}\right)-\theta^{\prime \prime} D_{x}(\xi),
\end{gathered}
$$


TABLE 10: Lie bracket of the admitted symmetry algebra for $m \neq n, n \neq-1$ and various $f(x)$.

\begin{tabular}{|c|c|c|c|c|c|c|}
\hline$f(x)=x^{a}$ & $a=0$ & $n$ arbitrary & $f(x)=x^{a}$ & $a=0$ & $n=-3 m-4$ & \\
\hline$\left[X_{i}, X_{j}\right]$ & $X_{1}$ & $X_{2}$ & {$\left[X_{i}, X_{j}\right]$} & $X_{1}$ & $X_{2}$ & $X_{3}$ \\
\hline$X_{1}$ & 0 & $X_{1}$ & $X_{1}$ & 0 & $X_{1}$ & $X_{3}$ \\
\hline \multirow[t]{2}{*}{$X_{2}$} & $X_{1}$ & 0 & $X_{2}$ & $-X_{1}$ & 0 & $2 X_{2}$ \\
\hline & & & $X_{3}$ & $-X_{3}$ & $-2 X_{2}$ & 0 \\
\hline$f(x)=x^{a}$ & $a=\frac{1}{2}$ & $n=-4 m-5$ & $f(x)=x^{a}$ & $a=1$ & $m=-1$ & \\
\hline$\left[X_{i}, X_{j}\right]$ & $X_{1}$ & $X_{2}$ & {$\left[X_{i}, X_{j}\right]$} & $X_{1}$ & $X_{2}$ & \\
\hline$X_{1}$ & 0 & $X_{1}$ & $X_{1}$ & 0 & $X_{1}$ & \\
\hline$X_{2}$ & $-X_{1}$ & 0 & $X_{2}$ & $-X_{1}$ & 0 & \\
\hline$f(x)=x^{a}$ & $a=2$ & $n=-m-2$ & $f(x)=e^{a x}$ & any $a$ & $n$ arbitrary & \\
\hline$\left[X_{i}, X_{j}\right]$ & $X_{1}$ & $X_{2}$ & {$\left[X_{i}, X_{j}\right]$} & $X_{1}$ & $X_{2}$ & \\
\hline$X_{1}$ & 0 & $X_{1}$ & $X_{1}$ & 0 & $X_{1}$ & \\
\hline$X_{2}$ & $-X_{1}$ & 0 & $X_{2}$ & $X_{1}$ & 0 & \\
\hline
\end{tabular}

TABLE 11: The type of second-order equations admitting $L_{2}$ for $m \neq n, n \neq-1$ and various $f(x)$.

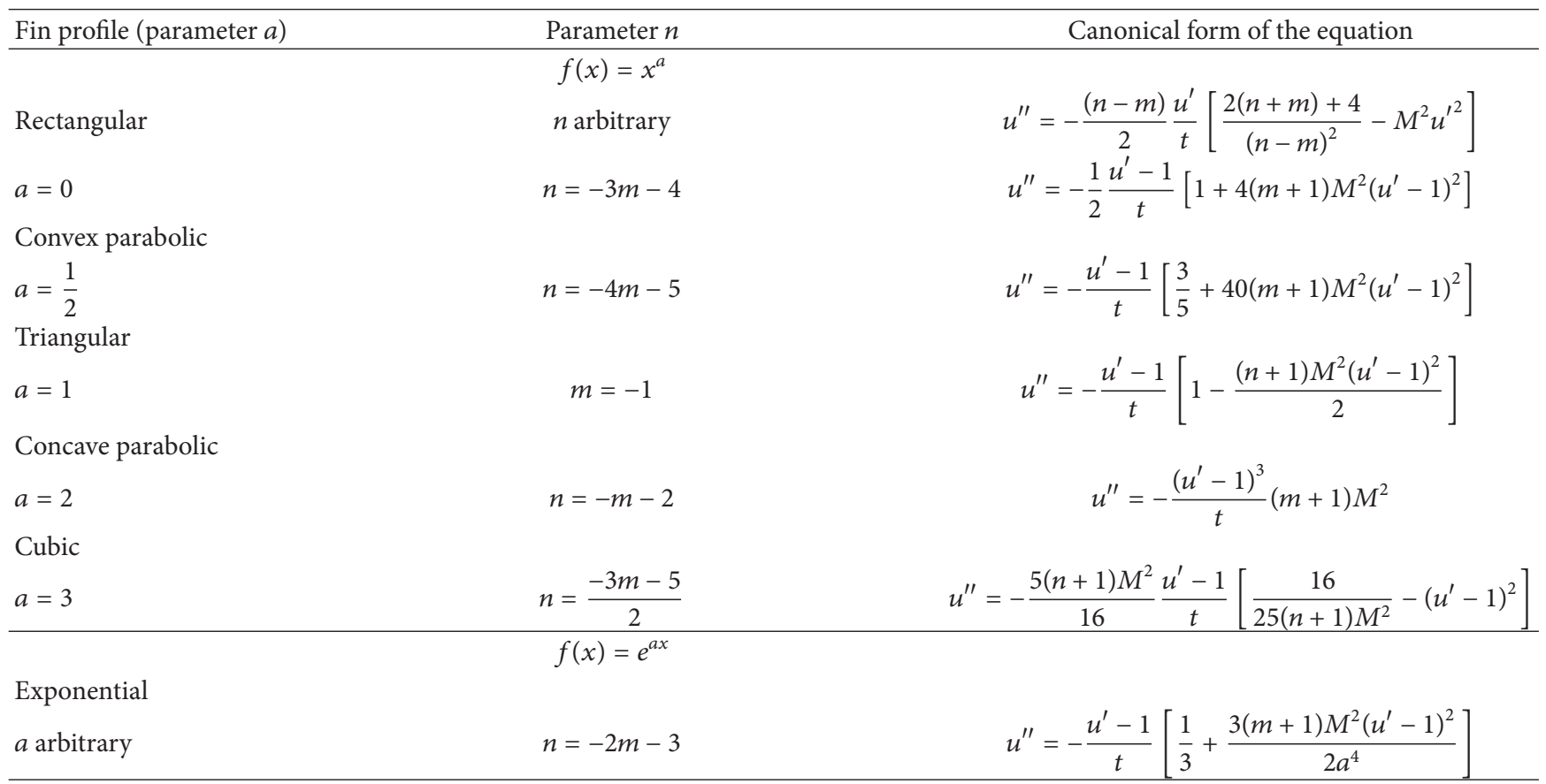

with $D_{x}$ being the total derivative operator defined by

$$
D_{x}=\partial_{x}+\theta^{\prime} \partial_{\theta}+\theta^{\prime \prime} \partial_{\theta^{\prime}}+\cdots
$$

The prime implies differentiation with respect to $\theta$. The invariance surface condition is given by

$$
X^{[2]}\left(\text { Equation (8)) }\left.\right|_{\text {Equation(8) }}=0 .\right.
$$

The coefficients of $X$ do not involve derivatives; we can separate (48) with respect to the derivatives of $\theta$ and solve the resulting overdetermined system of linear homogeneous partial differential equations known as determining equations. Further calculations were facilitated by the freely available package Dimsym [31], a subprogram of REDUCE [32].

\section{Symmetry Reductions and Invariant Solutions}

We employ the direct group classification to calculate the Lie point symmetries admitted by (8). A few cases arise.

5.1. Case: $m=n$. For $f(x)=x^{a}$ and $f(x)=e^{a x}$, the symmetries are listed in Table 9.

5.2. Case: $m \neq n, m \neq-1$, and $f(x)=(x+1)^{a}$

Example 7. As an illustrative example, we consider $f(x)=1$ and $n=-3 m-4$. The governing equation (8) becomes

$$
y^{\prime \prime}=(m+1) M^{2} y^{-3} \text {. }
$$


TABLE 12: Reductions arising from Table 9.

Fin profile (parameter $a)$

\section{Parameter $n$}

$$
f(x)=x^{a}
$$

Rectangular

$n=-3 m-4$

(i) $u^{\prime}=0 \Rightarrow u=$ const

$a=0$

(ii) $1+4(m+1) M^{2}\left(u^{\prime}-1\right)^{2}=0 \Rightarrow u=\left(1 \pm i \frac{1}{2 \sqrt{m+1} M}\right) t+c$

(iii) $u^{\prime \prime} \neq 0 \Rightarrow u=t \pm \frac{1}{\sqrt{m+1} M} \sqrt{c_{1} t-1}+c_{2}$

Convex parabolic

(i) $u^{\prime}=0 \Rightarrow u=$ const

$a=\frac{1}{2}$

$n=-4 m-5$

(ii) $\frac{3}{5}+40(m+1) M^{2}\left(u^{\prime}-1\right)^{2} \Rightarrow u=\left(1 \pm i \frac{1}{10 M} \sqrt{\frac{3}{2(m+1)}}\right) t+c$

(iii) $u^{\prime \prime} \neq 0 \Rightarrow u=t \pm \frac{1}{10 M} \sqrt{\frac{3}{2(m+1)}} \ln \left[t+\sqrt{c_{1} t^{2}-1}\right]+c_{2}$

Triangular

(i) $u^{\prime}=0 \Rightarrow u=$ const

$a=1$

$m=-1$

(ii) $1-\frac{(n+1) M^{2}\left(u^{\prime}-1\right)^{2}}{2}=0 \Rightarrow u=\left(1 \pm \frac{1}{M} \sqrt{\frac{2}{n+1}}\right) t+c$

(iii) $u^{\prime \prime} \neq 0 \Rightarrow u=t \pm M \sqrt{\frac{n+1}{2}} \arcsin c_{1} t+c_{2}$

Concave parabolic

$a=2$

$n=-m-2$

(i) $u^{\prime}=0 \Rightarrow u=$ const

Cubic

$a=3$

$$
m=\frac{-3 m-5}{2}
$$

(ii) $u^{\prime \prime} \neq 0 \Rightarrow u=t \pm \int \frac{1}{\sqrt{c+(m+1) M^{2} \ln t}} \mathrm{dt}$

(i) $u^{\prime}=0 \Rightarrow u=$ const

(ii) $\frac{16}{25(n+1) M^{2}}-\left(u^{\prime}-1\right)^{2} \Rightarrow u=\left(1 \pm \frac{4}{M \sqrt{n+1}}\right) t+c$

(iii) $u^{\prime \prime} \neq 0 \Rightarrow u=t \pm \frac{4}{M \sqrt{n+1}} \int \frac{\mathrm{dt}}{\sqrt{1-\left(c_{1} t\right)^{2 / 5}}}+c_{2}$

$$
f(x)=e^{a x}
$$

Exponential

$n=-2 m-3$

(i) $u^{\prime}=0 \Rightarrow u=$ const

(ii) $\frac{1}{3}+\frac{3(m+1) M^{2}\left(u^{\prime}-1\right)^{2}}{2 a^{4}} \Rightarrow u=\left(1 \pm i \frac{a^{2}}{3 M} \sqrt{\frac{2}{m+1}}\right) t+c$

(iii) $u^{\prime \prime} \neq 0 \Rightarrow u=t \pm \int \frac{a^{2}}{3 M} \sqrt{\frac{2}{m+1}} \frac{1}{\sqrt{\left(c_{1} t\right)^{2 / 3}-1}} \mathrm{dt}+c_{2}$

TABLE 13: Original variables for $m \neq n, n \neq-1$ and various $f(x)$.

Fin profile (parameter $a$ ) Parameter $n$

$f(x)=x^{a}$

Rectangular

$a=0$

$n=-3 m-4$

$\theta=\left[1+(m+1) M^{2}\left(x^{2}-1\right)\right]^{1 /(2(m+1))}$

Convex parabolic

$a=\frac{1}{2}$

$n=-4 m-5$

Triangular

$a=1$

$m=-1$

Concave parabolic

$a=2$

$n=-m-2$

$f(x)=e^{a x}$

Exponential

$a$ arbitrary

$n=-2 m-3$

\section{Solution}




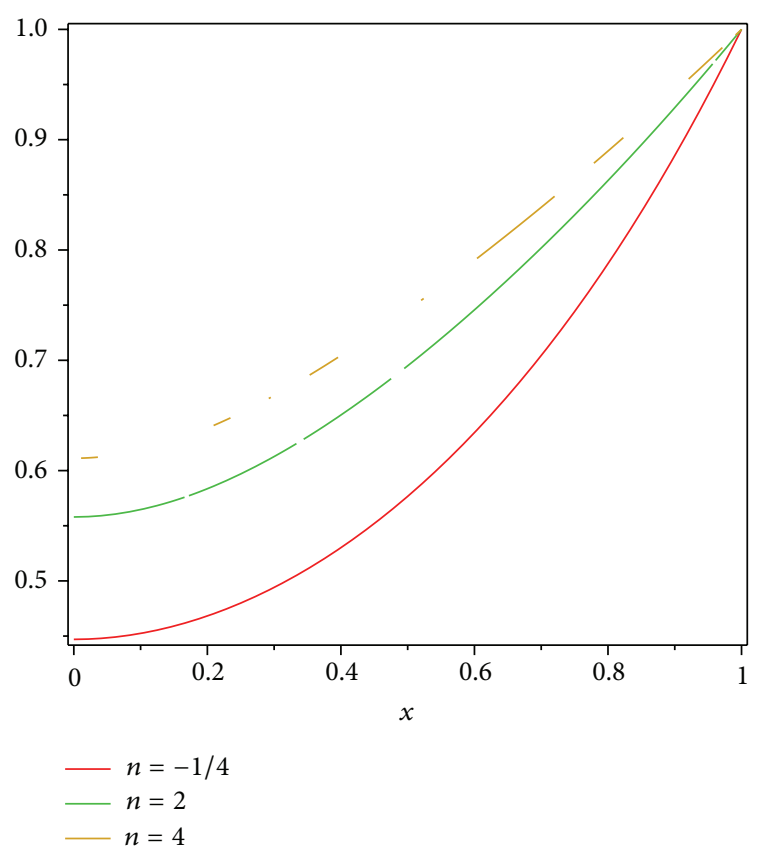

Figure 3: Temperature $f(x)=\sqrt{x+1}$ given in (18) in a fin with varying values of $n$.

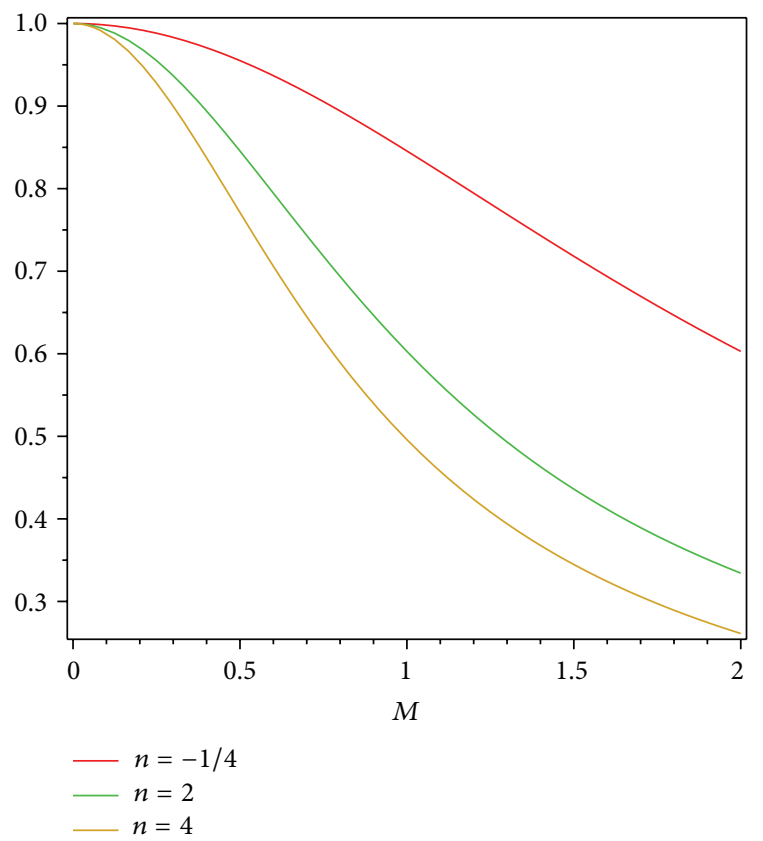

FIGURE 4: Efficiency $f(x)=\sqrt{x+1}$ as given in solution (21) in a fin with varying values of $n$.

We observe that (49) admits a non-Abelian twodimensional Lie algebra spanned by the base vectors listed in Table 9. This noncommuting pair of symmetries leads to the canonical variables

$$
t=y^{2}, \quad u=x+y^{2} .
$$

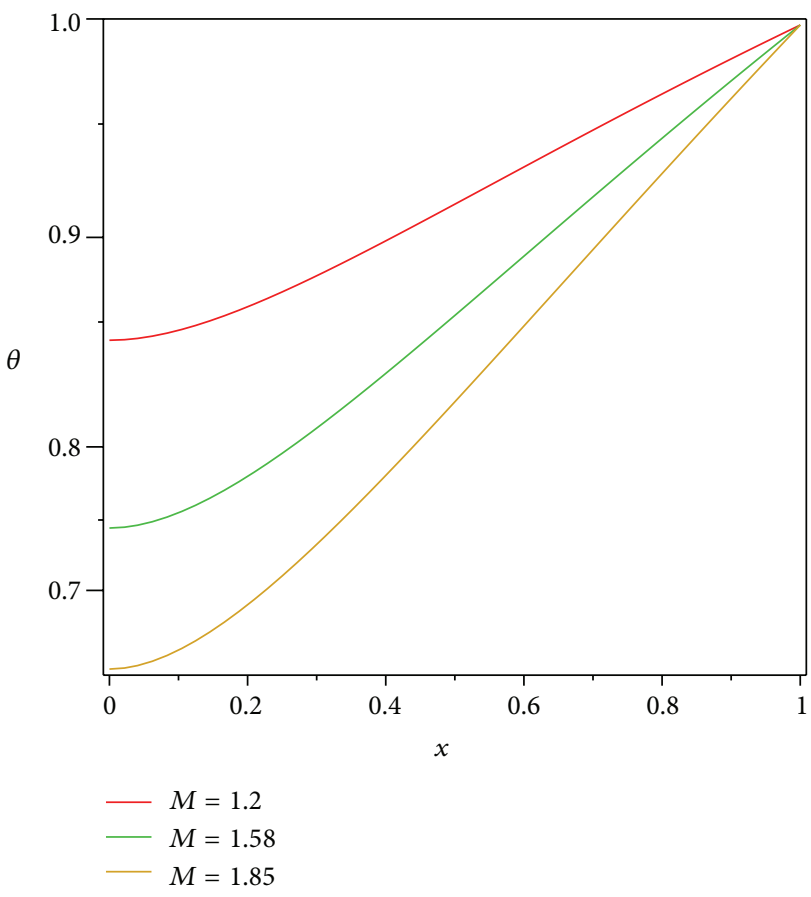

FIGURE 5: Temperature distribution of a fin profile $f(x)=(x+$ $1)^{3}$ as given in solution (22) in a fin with varying values of the thermogeometric fin parameter. Here, $n$ is fixed at $-1 / 4$.

The corresponding canonical forms of $X_{1}$ and $X_{2}$ are

$$
\Gamma_{1}=\partial_{u}, \quad \Gamma_{2}=t \partial_{t}+u \partial_{u}
$$

Writing $u=u(t)$ transforms (49) to

$$
u^{\prime \prime}=-\frac{1}{2} \frac{u^{\prime}-1}{t}\left[1+4(m+1) M^{2}\left(u^{\prime}-1\right)^{2}\right] .
$$

Here, the prime denotes the total derivative with respect to $t$. Three cases arise.

Subcase 1. For $u^{\prime}-1=0$, we obtain the constant which is not related to the original problem. Thus, we ignore it.

Subcase 2. If the term in the square bracket vanishes, then we obtain in terms of the original variables the exact "particular" solution

$$
\theta=[-1+i 2 \sqrt{m+1} M(x+1)]^{1 / 2(m+1)}
$$

which is not physically realistic. Therefore, we ignore this solution.

Subcase 3. Solving the entire equation (52), we obtain the two solutions that satisfy the boundary conditions. Consider the following:

$$
\theta=\left[1+(m+1) M^{2}\left(x^{2}-1\right)\right]^{1 / 2(m+1)} .
$$

The fin efficiency is given by

$$
\eta_{1}=\int_{0}^{1}\left[1+(m+1) M^{2}\left(x^{2}-1\right)\right]^{1 / 2} d x .
$$




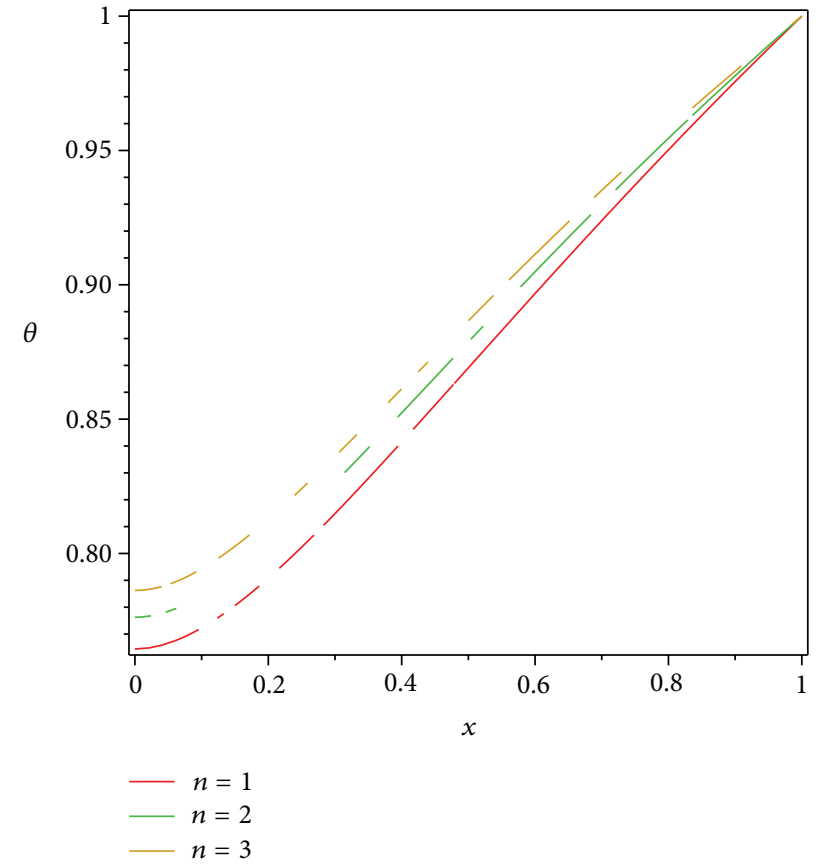

Figure 6: Temperature distribution of a fin profile $f(x)=(x+1)^{3}$ as given in solution (22) in a fin with varying values of $n$. Here, the thermogeometric fin parameter is fixed at 1.58.

Example 8. Given $f(x)=x+1$ and $n \neq m, m=-1$, then the governing equation (8) becomes

$$
y^{\prime \prime}=\frac{(n+1) M^{2} e^{y}-y^{\prime}}{x+1}
$$

after the substitution $e^{y}=\theta^{n+1}$. The two-dimensional Lie algebra admitted by (56) is listed in Table 9. These resulting canonical variables are

$$
t=\frac{1}{\sqrt{(x+1) e^{y}}}, \quad u=2-\ln (x+1)+\frac{1}{\sqrt{(x+1) e^{y}}} .
$$

The corresponding canonical forms of $X_{1}$ and $X_{2}$ are

$$
\Gamma_{1}=\partial_{u}, \quad \Gamma_{2}=t \partial_{t}+u \partial_{u}
$$

By writing $u=u(t),(56)$ is transformed into

$$
u^{\prime \prime}=-\frac{1}{t}\left(u^{\prime}-1\right)\left[\frac{(n+1) M^{2}}{2}\left(u^{\prime}-1\right)^{2}-1\right] .
$$

As in the previous example, three cases arise.

Subcase 4. For $u^{\prime}-1=0 \Rightarrow u=t+c-1 \Rightarrow 2-\ln (x+1)=$ $c_{1}$. This is not related to the original problem. Thus, it is not considered.

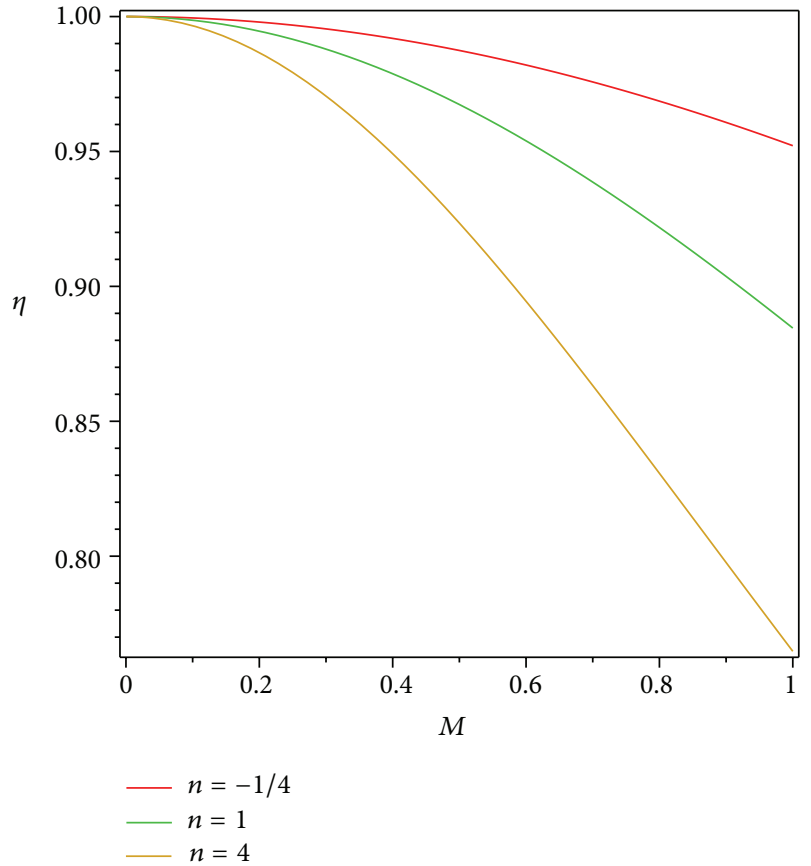

FIgURE 7: Fin efficiency for the profile $f(x)=(x+1)^{3}$ as given in solution (25) in a fin with varying values of $n$.

Subcase 5. If the term in the square bracket vanishes, then we obtain in terms of the original variables the exact "particular" solution

$$
\begin{aligned}
\theta=[2 \times( & (n+1) M^{2}(x+1) \\
& \left.\left.\times\left(\ln 2-\ln (x+1)+\frac{1}{M \sqrt{n+1}}\right)^{2}\right)^{-1}\right]^{1 /(n+1)}
\end{aligned}
$$

which satisfies the boundary condition only at one end.

Subcase 6. Lastly, we solve the entire equation (59) and obtain the solution that satisfies both the Dirichlet and the Neumann boundary conditions. Consider the following:

$$
\begin{gathered}
\theta=\left[2 \cosh ^{2}\left(\tanh ^{-1}\left(\frac{1}{2 M} \sqrt{\frac{2}{n+1}}\right)-M \sqrt{\frac{n+1}{2}} \ln 2\right)\right. \\
\times\left(( x + 1 ) \operatorname { c o s h } ^ { 2 } \left(\tanh ^{-1}\left(\frac{1}{2 M} \sqrt{\frac{2}{n+1}}\right)\right.\right. \\
\left.\left.\left.-M \sqrt{\frac{n+1}{2}} \ln (x+1)\right)\right)^{-1}\right]^{1 /(n+1)} .
\end{gathered}
$$




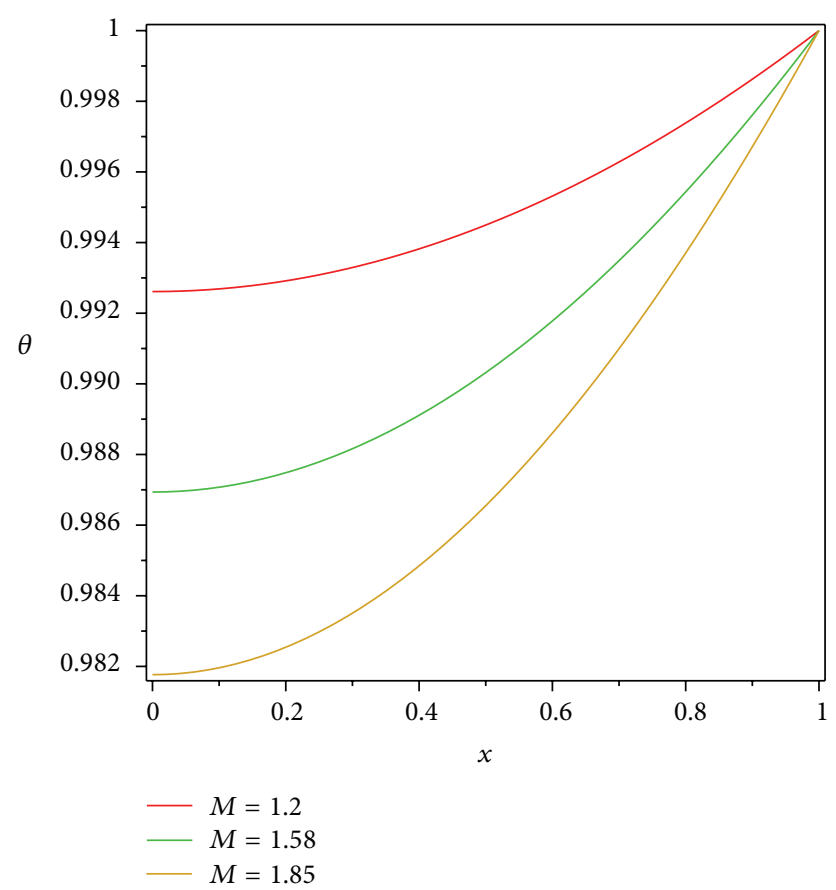

FIgURE 8: Temperature distribution for the profile $f(x)=1, n \neq m$ as given in solution (41) in a fin with varying values of $M$.

The fin efficiency is given by

$$
\begin{array}{r}
\eta_{1}=\int_{0}^{1}\left[2 \cosh ^{2}\left(\tanh ^{-1}\left(\frac{1}{2 M} \sqrt{\frac{2}{n+1}}\right)-M \sqrt{\frac{n+1}{2}} \ln 2\right)\right. \\
\times\left((x+1) \cosh ^{2}\right. \\
\times\left(\tanh ^{-1}\left(\frac{1}{2 M} \sqrt{\frac{2}{n+1}}\right)\right. \\
\left.\left.\left.-M \sqrt{\frac{n+1}{2}} \ln (x+1)\right)\right)^{-1}\right] d x .
\end{array}
$$

The temperature distribution along the surface for this profile is depicted in Figures 8 and 9. The fin efficiency as function of the thermogeometric fin parameter is shown in Figure 10.

\section{Some Discussions}

We now analyze fin problem using solutions given in (18) and (21). We observe in Figure 5 that, for the case of laminar film boiling or condensation, the temperature is inversely proportional to the thermogeometric fin parameter. An increase in values of $M$ yielded the decrease in values of temperature. Temperature distribution along the surface was studied for varying values of $n$, while $M$ was kept constant. The results depicted in Figure 6 show that the temperature is directly proportional to the parameter $n$. The fin efficiency

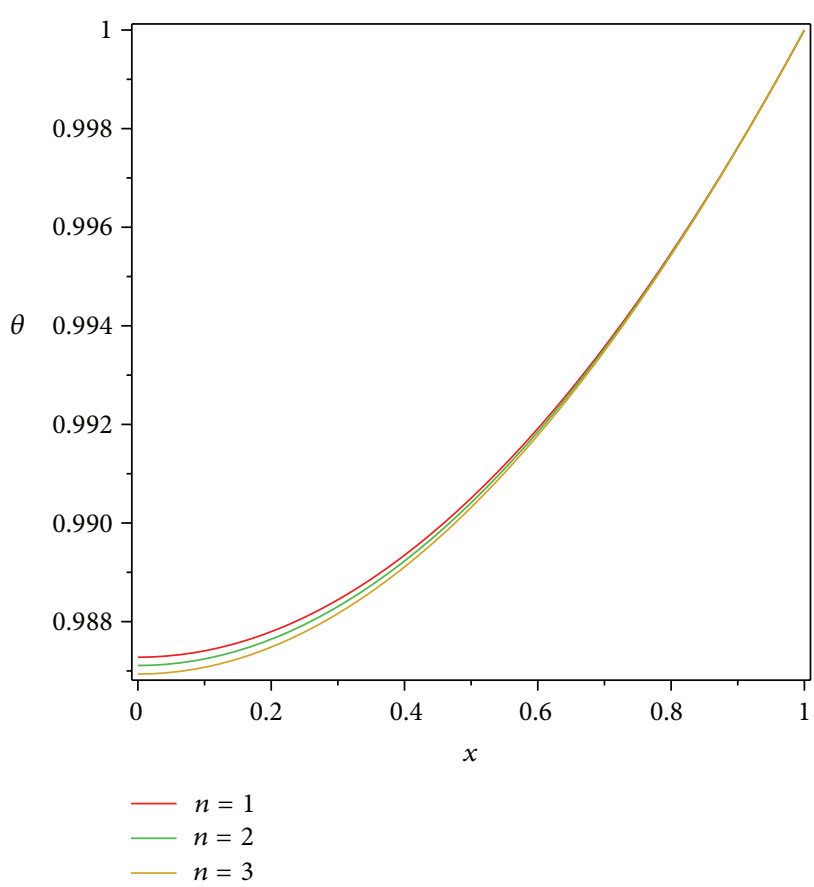

FIgURE 9: Temperature $f(x)=1, n \neq m$ as given in solution (41) in a fin with varying values of $n$.

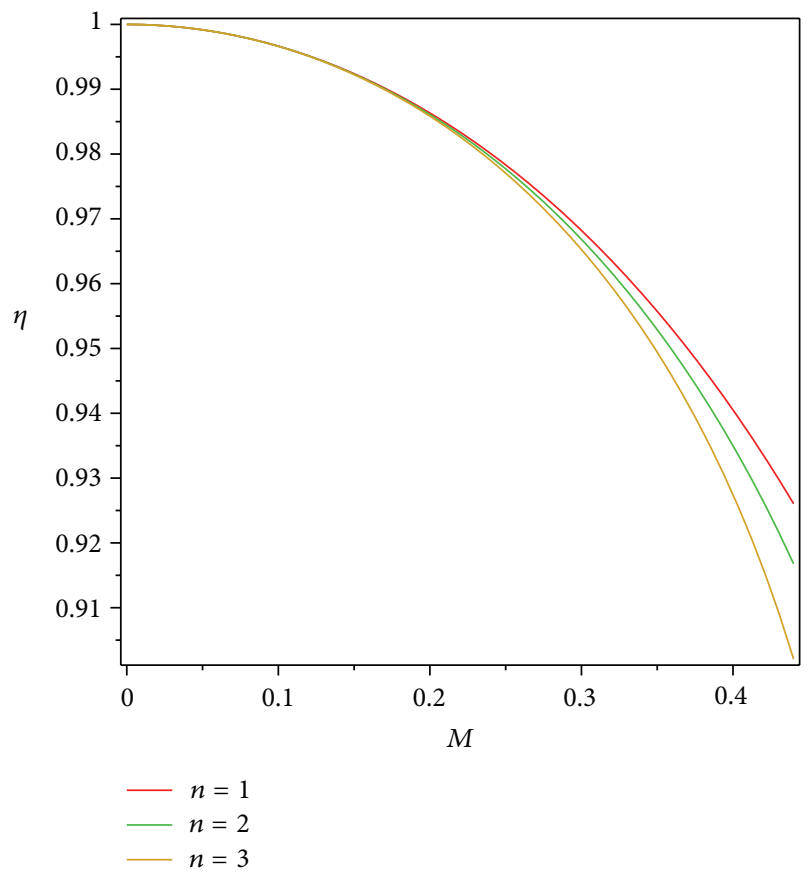

FIGURE 10: Efficiency $f(x)=1, n \neq m$ as given in solution (42) in a fin with varying values of $n$.

as function of the thermogeometric fin parameter is shown in Figure 7. Similar trends can be observed from the figures showing temperature distribution and efficiency for other profiles.

The Lie commutators or Lie brackets are given in Table 10 and further reductions are provided in Table 11. Solutions for 
$f(x)=x^{a}$ are furnished in Table 12. The solutions in terms of the original variables are listed in Table 13. Most of these exact solutions do not satisfy one of the boundary conditions. Symmetries and further analysis of $f(x) \in\{\sin x, \cos x, \ln x\}$ were ignored in this paper. The solution for $f(x)=e^{a x}$ for $n=m$ is given in [33]; therefore, we focused on the case where $n \neq m$.

\section{Concluding Remarks}

Exact solutions for fin problem with power law temperaturedependent thermal conductivity and heat transfer coefficient were constructed. Lie symmetry techniques were used in cases where direct integration was not feasible. Results showing longitudinal fin of various profiles were presented. The obtained solutions satisfy the physical boundary conditions. The exact solutions constructed here could be used as benchmarks or validation tests for numerical schemes.

\section{Conflict of Interests}

The authors declare that there is no conflict of interests regarding the publication of this paper.

\section{Acknowledgments}

M. D. Mhlongo would like to thank the Defence, Peace, Safety and Security Landward Sciences in the Council for Scientific and Industrial Research and the University of the Witwatersrand for financial support. R. J. Moitsheki wishes to thank the National Research Foundation of South Africa for the generous financial support.

\section{References}

[1] A. D. Kraus, A. Aziz, and J. Welty, Extended Surface Heat Transfer, John Wiley \& Sons, New York, NY, USA, 2001.

[2] F. Khani, M. A. Raji, and H. H. Nejad, "Analytical solutions and efficiency of the nonlinear fin problem with temperaturedependent thermal conductivity and heat transfer coefficient," Communications in Nonlinear Science and Numerical Simulation, vol. 14, no. 8, pp. 3327-3338, 2009.

[3] F. Khani, M. Ahmadzadeh Raji, and S. Hamedi-Nezhad, "A series solution of the fin problem with a temperature-dependent thermal conductivity," Communications in Nonlinear Science and Numerical Simulation, vol. 14, no. 7, pp. 3007-3017, 2009.

[4] S. Kim and C.-H. Huang, "A series solution of the non-linear fin problem with temperature-dependent thermal conductivity and heat transfer coefficient," Journal of Physics D: Applied Physics, vol. 40, no. 9, pp. 2979-2987, 2007.

[5] A. Aziz and T. Y. Na, "Periodic heat transfer in fins with variable thermal parameters," International Journal of Heat and Mass Transfer, vol. 24, no. 8, pp. 1397-1404, 1981.

[6] F. Khani and A. Aziz, "Thermal analysis of a longitudinal trapezoidal fin with temperature-dependent thermal conductivity and heat transfer coefficient," Communications in Nonlinear Science and Numerical Simulation, vol. 15, no. 3, pp. 590-601, 2010.
[7] S. Kim and C.-H. Huang, "A series solution of the fin problem with a temperature-dependent thermal conductivity," Journal of Physics D: Applied Physics, vol. 39, no. 22, pp. 4894-4901, 2006.

[8] R. J. Moitsheki and A. Rowjee, "Steady heat transfer through a two-dimensional rectangular straight fin," Mathematical Problems in Engineering, vol. 2011, Article ID 826819, 13 pages, 2011.

[9] P. L. Ndlovu and R. J. Moitsheki, "Application of the twodimensional transform method to heat conduction problem for heat transfer in longitudinal rectangular and convex parabolic fins," Communication in Nonlinear Science and Numerical Simulation, vol. 18, no. 10, pp. 2689-2698, 2013.

[10] R. J. Moitsheki, T. Hayat, and M. Y. Malik, "Some exact solutions of the fin problem with a power law temperature-dependent thermal conductivity," Nonlinear Analysis: Real World Applications, vol. 11, no. 5, pp. 3287-3294, 2010.

[11] R. J. Moitsheki and M. K. Banda, "Heat transfer in a convective straight fin with temperature dependent thermal conductivity and heat transfer coefficient," in Proceedings of the Interntional Conference on Mathematical Application in Engineering, pp. 388-392, Kuala Lumpur, Malasya, 2010.

[12] A. H. Bokhari, A. H. Kara, and F. D. Zaman, "A note on a symmetry analysis and exact solutions of a nonlinear fin equation," Applied Mathematics Letters, vol. 19, no. 12, pp. 13561360, 2006.

[13] M. Pakdemirli and A. Z. Sahin, "Group classification of fin equation with variable thermal properties," International Journal of Engineering Science, vol. 42, no. 17-18, pp. 1875-1889, 2004.

[14] M. Pakdemirli and A. Z. Sahin, "Similarity analysis of a nonlinear fin equation," Applied Mathematics Letters, vol. 19, no. 4, pp. 378-384, 2006.

[15] O. O. Vaneeva, A. G. Johnpillai, R. O. Popovych, and C. Sophocleous, "Group analysis of nonlinear fin equations," Applied Mathematics Letters, vol. 21, no. 3, pp. 248-253, 2008.

[16] R. O. Popovych, C. Sophocleous, and O. O. Vaneeva, "Exact solutions of a remarkable fin equation," Applied Mathematics Letters, vol. 21, no. 3, pp. 209-214, 2008.

[17] R. J. Moitsheki and C. Harley, "Transient heat transfer in longitudinal fins of various profiles with temperature-dependent thermal conductivity and heat transfer coefficient," Pramana, vol. 77, no. 3, pp. 519-532, 2011.

[18] R. J. Moitsheki and M. D. Mhlongo, "Classical lie point symmetry analysis of a steady nonlinear one-dimensional fin problem," Journal of Applied Mathematics, vol. 2012, Article ID 671548, 13 pages, 2012.

[19] K. Abu-Abdou and A. A. M. Mujahid, "Heat transfer in straight fins of arbitrary profile subjected to periodic base and environment temperatures," Wärme- und Stoffübertragung, vol. 24, no. 6, pp. 353-361, 1989.

[20] E. Assis and H. Kalman, "Transient temperature response of different fins to step initial conditions," International Journal of Heat and Mass Transfer, vol. 36, no. 17, pp. 4107-4114, 1993.

[21] M. D. Mhlongo, R. J. Moitsheki, and O. D. Makinde, “Transient response of longitudinal rectangular fins to step change in base temperature and in base heat flow conditions," International Journal of Heat and Mass Transfer, vol. 57, pp. 117-125, 2013.

[22] H. C. Ünal, "The effect of the boundary condition at a fin tip on the performance of the fin with and without internal heat generation," International Journal of Heat and Mass Transfer, vol. 31, no. 7, pp. 1483-1496, 1988.

[23] K. Laor and H. Kalman, "Performance and optimum dimensions of different cooling fins with a temperature-dependent 
heat transfer coefficient," International Journal of Heat and Mass Transfer, vol. 39, no. 9, pp. 1993-2003, 1996.

[24] A. D. Polyanin and V. F. Zaitsev, Handbook of Exact Solutions for Ordinary Differential Equations, Chapman and Hall, CRC Press, Boca Raton, Fla, USA, 2nd edition, 2003.

[25] G. W. Bluman and S. Anco, Symmetry and Integration Methods for Differential Equations, Springer, New York, NY, USA, 2002.

[26] G. W. Bluman and S. Kumei, Symmetries and Integration Methods for Differential Equations, Springer, New York, NY, USA, 1989.

[27] N. H. Ibragimov, Elementary Lie Group Analysis and Ordinary Differential Equations, John Wiley \& Sons, 1998.

[28] P. J. Olver, Applications of Lie Groups to Differential Equations, Springer, New York, NY, USA, 1993.

[29] L. V. Ovisianikov, Group Analysis of Differential Equations, Academic Press, New York, NY, USA, 1982.

[30] N. H. Ibragimov, M. Torrisi, and A. Valenti, "Preliminary group classification of equations $v_{t} t=f\left(x, v_{x}\right) v_{x x}+g\left(x, v_{x}\right)$," Journal of Mathematical Physics, vol. 32, no. 11, pp. 2988-2995, 1991.

[31] J. Sherring, Dimsym: Symmetry Determination and Linear Differential Equation Package, Mathematics Department, La Trobe University, Melbourne, VIC, Australia, 1993.

[32] A. C. Hearn, REDUCE User's Manual Version 3.8, Rand Corporation Publication CP78, Santa Monica, Calif, USA, 1985.

[33] M. Turkyilmazoglu, "Exact solutions to heat transfer in straight fins of varying exponential shape having temperature dependent properties," International Journal of Thermal Sciences, vol. 55, pp. 69-75, 2012. 


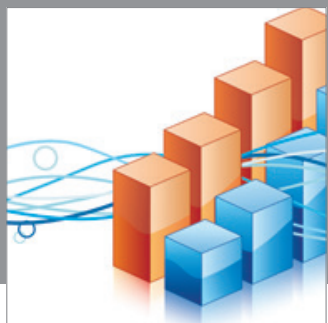

Advances in

Operations Research

mansans

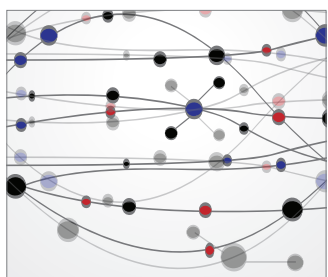

The Scientific World Journal
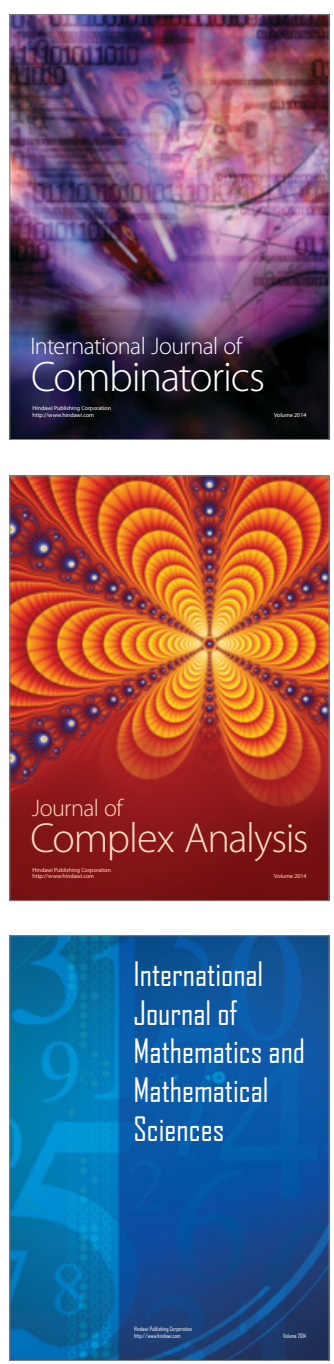
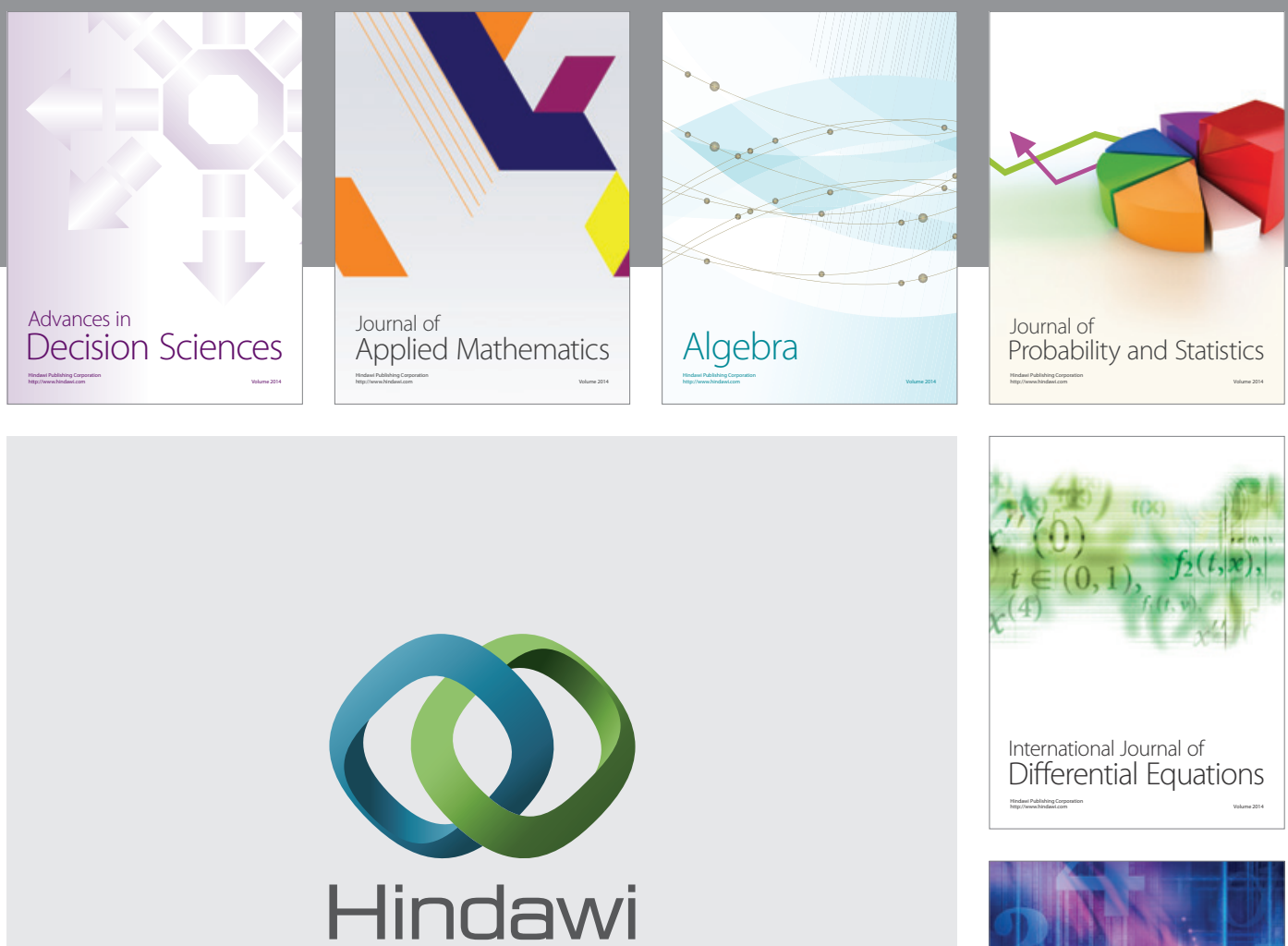

Submit your manuscripts at http://www.hindawi.com
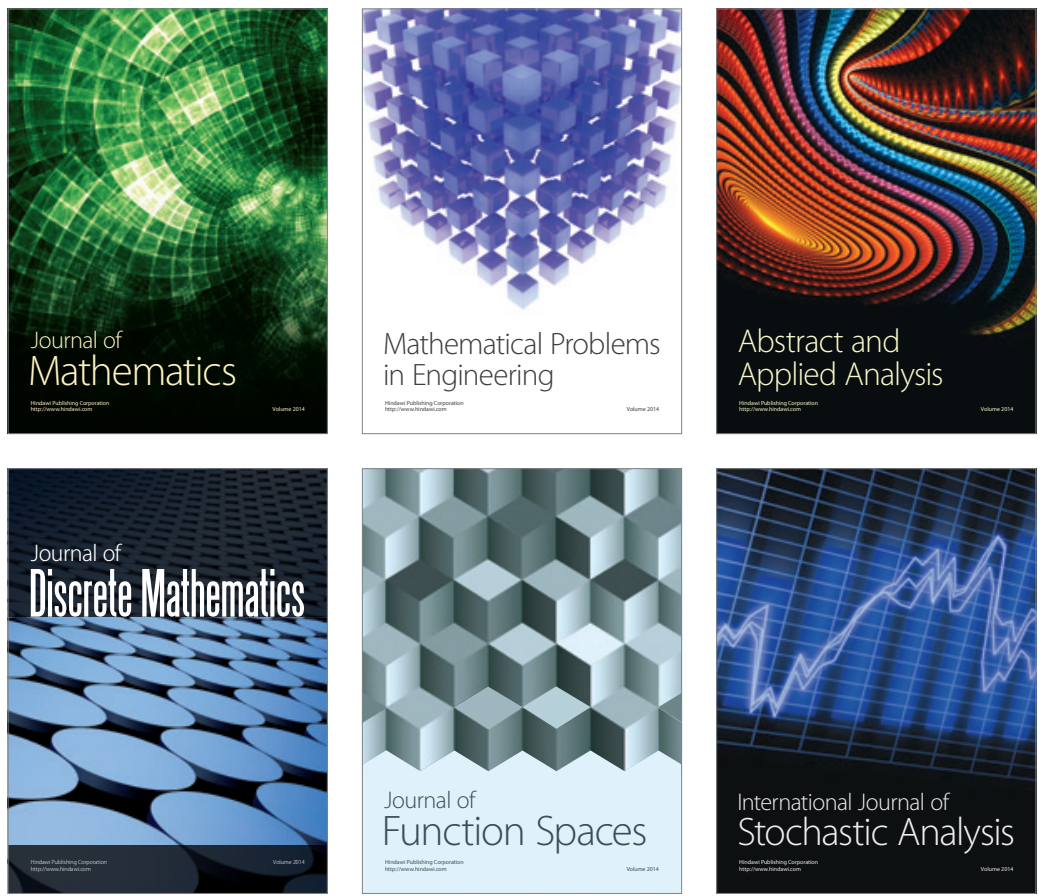

Journal of

Function Spaces

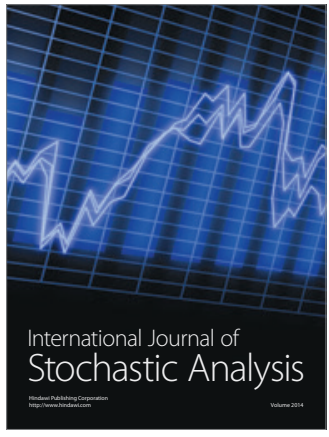

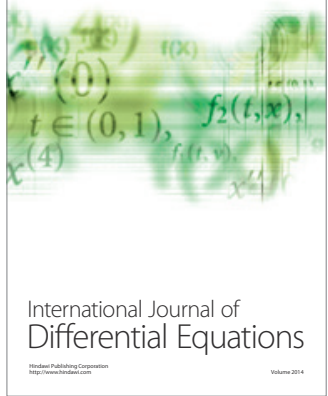
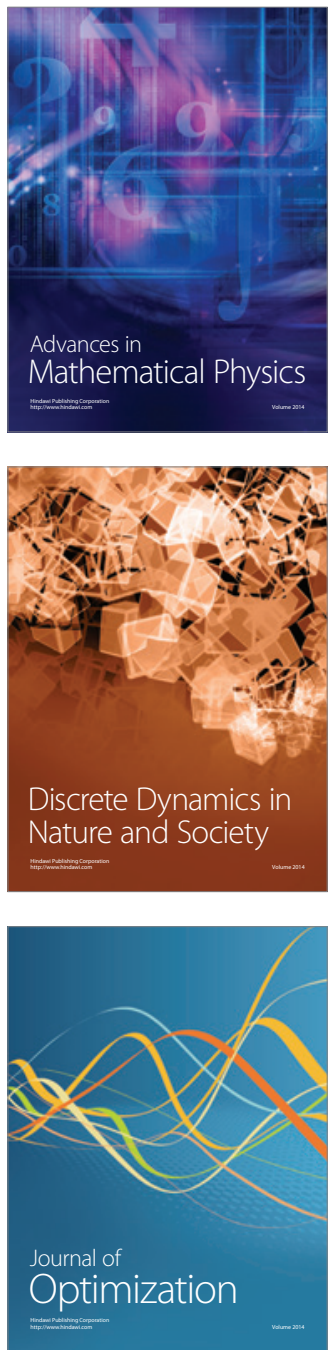